\title{
A CHARACTERIZATION OF COMMUTATIVE GROUP ALGEBRAS AND MEASURE ALGEBRAS
}

\author{
BY \\ MARC A. RIEFFEL $\left({ }^{1}\right)$
}

Let $G$ be a locally compact Abelian group. By the group algebra, $L(G)$, of $G$ we mean the Banach algebra of all (equivalence classes of) complexvalued functions on $G$ integrable with respect to the Haar measure on $G$, with convolution as multiplication. By the measure algebra, $M(G)$, of $G$ we mean the Banach algebra of all finite complex-valued regular Borel measures on $G$, with convolution as multiplication.

In this paper we give a characterization of those commutative Banach algebras which are the group algebras of locally compact Abelian groups, and we give a characterization of those commutative Banach algebras which are the measure algebras of locally compact Abelian groups. Our results have been announced already, without proofs, in [13].

We will now give a brief outline of our results. In $\$ 1$ we define an abstract complex $L$-space, and, in extension of Kakutani's theorem [11] on the concrete representation of abstract real $L$-spaces, we show that any abstract complex $L$-space is isometrically isomorphic to complex $L^{1}(X, m)$ for some measure space $(X, m)$.

Let $B$ be any Banach space and $f$ any nonzero linear functional on $B$. Let $P(f)=\{x: f(x)=\|f\|\|x\|\}$. Then $P(f)$ is a cone in $B$ and so defines a partial order on $B$ in the usual way. In $\$ 2$ we study this phenomenon briefly, with particular attention to the case in which $B$ under this order becomes an abstract complex $L$-space.

Now let $A$ be any complex Banach algebra, with multiplication denoted by $*$, and let $f$ be a multiplicative linear functional (m.l.f.) on $A$. We say that $f$ is $L^{\prime}$-inducing if, under the order defined by $P(f), A$ is an abstract complex $L$-space, and if two other hypotheses are satisfied. In $\$ 3$ we see that if $f$ is any m.l.f. of $L(G)$ or $M(G)$ corresponding to a (continuous) character of $G$, then $f$ is $L^{\prime}$-inducing.

Our characterization of group algebras is a partial converse of this observation:

Theorem A. Let $A$ be a commutative semisimple Banach algebra such that

(1) Every m.l.f. of $A$ is $L^{\prime}$-inducing,

(2) $A$ is Tauberian.

Received by the editors March 23, 1964.

(1) Part of the research for this paper was conducted while the author held a National Science Foundation Summer Fellowship for Graduate Teaching Assistants. 
Then there exists a locally compact Abelian group, $G$, such that $A$ is isometrically isomorphic to $L(G)$.

Condition (2) means that the elements of $A$ whose Gelfand transforms have compact support are dense in $A$. In $\$ 5$ we give an example to show that condition (2) is necessary.

Part of the proof of Theorem A can be given the following more general setting:

Theorem B. Let $A$ be a complex commutative Banach algebra, and let $D$ be the collection of all $L^{\prime}$-inducing m.l.f. of $A$. Suppose that

(1) $D$ is a separating family of linear functionals for $A$. Then there exists a compact group, $B$, such that $A$ can be embedded in $M(B)$.

Suppose in addition that

(2) $D$ is locally compact in the $w^{*}$ topology. Then there exists a locally compact group, $G$, whose Bohr compactification is $B$ and whose dual group is homeomorphic to $D$, such that $A$ can be embedded in $M(B)$.

The proof of Theorem $B$ is given in $\$ 4$, and the rest of the proof of Theorem $A$ is given in $\$ 6$. Finally, $\$ 7$ is devoted to a characterization of measure algebras using Theorem $B$.

Perhaps the most surprising feature of our characterizations is that the involution which is usually defined in group algebras plays no role in our work. Also we do not assume at any point that the Banach algebras involved have any kind of approximate identity.

The contents of this paper are contained in a dissertation submitted in partial fulfillment of the requirements for the Ph.D. at Columbia University. It is with great pleasure that we express here our gratitude to Professor Richard V. Kadison for his encouragement of this work and for innumerable helpful comments and suggestions.

It is also a pleasure to acknowledge our indebtedness to Professor Andrew M. Gleason for showing us how to prove a result asserting the existence of nontrivial semicharacters on semigroups embedded in groups, which resulted in a considerable simplification of a proof. This result (Theorem 6.4) is of some independent interest.

We are also pleased to record our indebtedness to E. G. Effros and R. M. Dudley for reasons which will be acknowledged more specifically below.

1. Complex $L$-spaces. G. Birkhoff defines an abstract $L$-space [2, p. 254] to be a real Banach lattice such that:

(I) If $x \geqq 0$ and $y \geqq 0$ then $\|x+y\|=\|x\|+\|y\|$.

These spaces were first introduced by Birkhoff in [3]. Kakutani [11] studies abstract $L$-spaces which also satisfy:

(II) If $x \wedge y=0$ then $\|x+y\|=\|x-y\|$. 
In view of the following theorem which Kakutani proves in [11], whenever we speak of an abstract $L$-space in the sequel we will always assume that it also satisfies axiom (II).

1.1. Theorem. (our own phrasing) Let $B$ be an abstract L-space (satisfying axiom (II)). Then there exists a totally disconnected locally compact topological space, $X$, which is the direct sum of a (possibly uncountable) family $\left\{X_{\alpha}\right\}_{\alpha \in A}$ of totally disconnected compact spaces, and there exists a regular positive Borel measure, $m$, on $X$, such that $B$ is isometrically linear and order isomorphic to real $L(X, m)$.

Here, as in the sequel, by $L(X, m)$ we mean $L^{1}(X, m)$. By direct sum, we mean in the sense of categories. What this amounts to is that $X$ is the disjoint union of the $X_{\alpha}$ and, further, that a subset, $S$, of $X$ is open in $X$ if and only if the intersection of $S$ with $X_{\alpha}$ is open in $X_{\alpha}$ for each $\alpha$.

Because the complex numbers are a more natural field of scalars than the real numbers for the study of group algebras, we find it necessary to extend the definition of abstract $L$-spaces to Banach spaces over the complex numbers, and to prove the obvious extension of Kakutani's theorem. This extension proceeds as would be expected except for a detail which is analogous to the difficulties encountered with the norm when complexifying a real Banach algebra.

To begin with, an abstract complex $L$-space is to be a complex Banach space which is also a partially ordered vector space satisfying axiom (I). Further, if we define

$$
\begin{aligned}
& P=\{x: x \in B \text { and } x \geqq 0\}, \\
& R=\{x-y: x, y \in P\},
\end{aligned}
$$

then we require that:

(III) $P$ is norm closed in $B$.

(IV) $P$, with the order of $B$ restricted to $P$, is a lattice.

By Proposition 8 of [5, Chapter VI, p. 13] axiom (IV) implies that $R$ is a vector lattice under the order of $B$ restricted to $R$. Next we require that the following form of axiom (II) should hold:

(V) If $x, y \in R$ and $x \wedge y=0$, then $\|x+y\|=\|x-y\|$.

In the sequel, it will be important to know the following fact, which we prove here to show that it is independent of the further axioms which we will impose on $B$.

1.2. Proposition. $R$ is closed in $B$ (and so is a real abstract L-space).

Proof. We need the following relations for $x, y \in R$

$$
\left\|x^{+}-y^{+}\right\| \leqq\|x-y\| \text { and }\left\|x^{-}-y^{-}\right\| \leqq\|x-y\| \text {. }
$$


These rrlations follow immediately from Kakutani's Lemma 3.1 [11], the prof of wh goes through without change in our slightly more general setting.

Then if $x_{n}$ is a Cauchy sequence in $R$, by (1.2.1) $x_{n}^{+}$and $x_{n}^{-}$are Cauchy sequences in $P$, and so, since $P$ is closed, converge to points $y, z$ of $P$, respectively. Then if we let $x=y-z$, it is clear that $x$ is in $R$, and $x_{n}$ converges to $x$.

The next axiom which we require $B$ to satisfy is:

(VI) For any $x$ in $B$ there exist unique $y, z$ in $R$ such that $x=y+i z$ $(i=\sqrt{ }-1)$. (We will often write $y=\operatorname{Re}(x), z=\operatorname{Im}(x)$.)

It can be easily shown that axiom $(\mathrm{X})$ is independent of the earlier axioms.

Finally, we need to consider the norm in $B$. In a concrete complex $L$ space, given any element $x=y+i z$ where $y, z$ are real, one defines

$$
|x|=\left(y^{2}+z^{2}\right)^{1 / 2} \text {. }
$$

(Throughout this paper we will not distinguish between elements of $L(X, m)$ and their representative functions except where this is important. The same applies to $L^{\infty}(X, m)$.) Then every concrete $L$-space has the property that

$$
\|x\|=\||x|\| \text { for all } x
$$

(for a real abstract $L$-space this property follows from axioms (I) and (II), using the definition $|x|=x \bigvee(-x)$ ). Now (1.3) has no apparent formulation in an abstract complex $L$-space. But we need to use property (1.4) in order to insure that the norm behaves properly in our extension of Kakutani's theorem. What we need to do is to give an alternative definition of || which can be formulated in abstract complex $L$-spaces. Now, since $R$ is a real abstract $L$-space, it is (boundedly) lattice complete, as is any real concrete $L$-space $[2, \mathrm{p} .249]$. Then if $x$ is in $B$, or in any concrete complex $L$-space, and if $x=y+i z$ where $x, y$ are in $R$, then for any real number $\theta$,

$$
\operatorname{Re}\left(e^{i \theta} x\right)=y \cos \theta-z \sin \theta \leqq|y|+|z|
$$

(where for $w$ in $R,|w|=w \bigvee(-w)$ ). Thus

$$
\bigvee\left\{\operatorname{Re}\left(e^{i \theta} x\right): \theta \in[0,2 \pi]\right\}
$$

is a well-defined element of $R$, and we now show that we can use this element as our definition of $|x|$.

1.5. Proposition. Let $L(X, m)$ be a concrete complex $L$-space, and let $x \in L(X, m)$, with $x=y+i z$, where $y, z$ are real. Then

$$
|x|=\left(y^{2}+z^{2}\right)^{12}=\bigvee\left\{\operatorname{Re}\left(e^{i t} x\right): \theta \in[0,2 \pi]\right\} .
$$

Proof. Let $\tilde{y}$ and $\tilde{z}$ be everywhere real representatives of $y$ and $z$, so that 
$\tilde{x}=\tilde{y}+i \tilde{z}$ is a representative of $x$. Then

$$
\left(\tilde{y}^{2}+\tilde{z}^{2}\right)-(\tilde{y} \cos \theta-\tilde{z} \sin \theta)^{2}=(\tilde{y} \sin \theta+\tilde{z} \cos \theta)^{2} \geqq 0,
$$

so that $\tilde{y} \cos \theta-\tilde{z} \sin \theta \leqq\left(\tilde{y}^{2}+\tilde{z}^{2}\right)^{1 / 2}$. But $\tilde{y} \cos \theta-\tilde{z} \sin \theta$ is a representative of $\operatorname{Re}\left(e^{i \theta} x\right)$, and so $\operatorname{Re}\left(e^{i \theta} x\right) \leqq|x|$ for all $\theta$. Thus $\bigvee\left\{\operatorname{Re}\left(e^{i \theta} x\right): \theta \in[0,2 \pi]\right\}$ $\leqq|x|$.

But if for any $s$ in $X$ we set $\theta_{0}=-\arg \tilde{x}(s)$, then $\tilde{y}(s) \sin \theta_{0}+\tilde{z}(s) \cos \theta_{0}$ $=0$, so that we can find, for any preassigned $\epsilon>0$, a rational $\theta_{1}$ such that ||$\tilde{x}\left|(s)-\operatorname{Re}\left(e^{i \theta_{1}} \tilde{x}\right)(s)\right|<\epsilon$. It is thus apparent that $|\tilde{x}|$ is the pointwise supremum of the $\operatorname{Re}\left(e^{i \theta} \tilde{x}\right)$ as $\theta$ ranges over $[0,2 \pi] \cap Q$, where $Q$ is the set of rational numbers. Thus, since by restricting $\theta$ to rational numbers we are in a countable situation, we can apply Proposition 6 of $[6$, Chapter $V$, p. 46] to obtain the fact that $|\tilde{x}|$ is a representative of

$$
\bigvee\left\{\operatorname{Re}\left(e^{i \theta} x\right): \theta \in[0,2 \pi] \cap Q\right\}
$$

Thus

$$
\begin{aligned}
|x| & \geqq \bigvee\left\{\operatorname{Re}\left(e^{i \theta} x\right): \theta \in[0,2 \pi]\right\} \\
& \geqq \bigvee\left\{\operatorname{Re}\left(e^{i \theta} x\right): \theta \in[0,2 \pi] \cap Q\right\}=|x| .
\end{aligned}
$$

1.6. Corollary. Let $X$ be a locally compact Hausdorff space, and let $M(X)$ be the Banach vector-lattice of all finite regular Borel measures on $X$. Then if $m$ is in $M(X)$, then

$$
|m|=\bigvee\left\{\operatorname{Re}\left(e^{i \theta} m\right): \theta \in[0,2 \pi]\right\} .
$$

Proof. $L(X,|m|)$ is a Banach vector-sublattice of $M(X)$, so we can apply the above proposition to $m$ as an element of $L(X,|m|)$.

Now that we know how to define $|x|$ in an abstract complex Banach space $B$ satisfying axioms (I) and (III)-(VI), the final axiom which we require $B$ to satisfy if it is to be an abstract complex $L$-space is:

(VII) $\|x\|=\||x|\|$ for every $x$ in $B$.

That axiom (VII) is independent of axioms (I), (III)-(VI) is shown by the following example which R. M. Dudley found for us. Let $B$ be the usual complex two-dimensional coordinate space, with its usual order, and with the following norm: If $z=\left(z_{1}, z_{2}\right)$, then

$$
\|z\|=\max \left\{\left|z_{1}+z_{2}\right|,\left|z_{1}-z_{2}\right|\right\} .
$$

Then it is easily checked that $B$ satisfies axioms (I), (III)-(VI). However, if $z=(1, i)$, so that $|z|=(1,1)$, then $\|z\|=\sqrt{ } 2$ whereas $\||z|\|=2$.

We are now in a position to prove the obvious extension of Kakutani's theorem for abstract complex $L$-spaces.

1.7. Theorem. Let $B$ be an abstract complex L-space. Then there exists a totally disconnected locally compact Hausdorff space, $X$, which is the direct 
sum of a (possibly uncountable) family $\left\{X_{\alpha}\right\}_{\alpha \in A}$ of totally disconnected comact "paces. and there exists a regular Borel measure, $m$, on $X$, such that $B$ is isometrically linear and order isomorphic to complex $L(X, m)$.

Proof. As we saw in 1.2 , the real subspace $R$ of $B$ is an abstract real $L$-space, and so we can apply Kakutani's theorem to $R$ and obtain $X$ and $m$ satisfying the desired properties, and such that $R$ is isometrically linear and order isomorphic to real $L(X, m)$. We denote this isomorphism by $K$. Then if $x$ is in $B$ we can extend $K$ to all of $B$ by defining $K(x)=K(\operatorname{Re}(x))$ $+i K(\operatorname{Im}(x))$. From axiom (VI) it is clear that this extended $K$ is a vector space and order isomorphism of $B$ onto complex $L(X, m)$. Finally, we must check that $K$ is an isometry. But, since $K$ is an order isomorphism, we have for $x$ in $B$,

$$
\begin{aligned}
K(|x|) & =K\left(\bigvee\left\{\operatorname{Re}\left(e^{i \theta} x\right): \theta \in[0,2 \pi]\right\}\right) \\
& =\bigvee\left\{\operatorname{Re}\left(e^{i \theta} K(x)\right): \theta \in[0,2 \pi]\right\}=|K(x)| .
\end{aligned}
$$

Then using axiom (VII)

$$
\|K(x)\|=\||K(x)|\|=\|K(|x|)\|=\||x|\|=\|x\| .
$$

We now consider the dual of an abstract $L$-space, $B$. From the fact that the $X$ obtained in the above representations is the direct sum of compact spaces $[12,15 \mathrm{D}]$, or $[8$, p. 290$]$, it follows that the dual of $B$ can be represented by $L^{\infty}(X, m)$ in the obvious way.

We will always take the dual pairing between $L(X, m)$ and $L^{\infty}(X, m)$, even in the complex case, to be defined by

$$
f(x)=\langle x, f\rangle=\int x f d m,
$$

where $x$ is in $L(X, m)$ and $f$ is in $L^{\infty}(X, m)$.

In several places in the sequel it will be useful to have the following proposition, which R. V. Kadison suggested to us.

1.8. Proposition. Let $B$ and $C$ be abstract or concrete complex $L$-spaces, and let $F$ be a linear isometric injection of $B$ into $C$, such that $F(x) \geqq 0$ if and only if $x \geqq 0$. Then

(1) $F(x)$ is real if and only if $x$ is real.

(2) $\operatorname{Re}(F(x))=F(\operatorname{Re}(x))$ and $\operatorname{Im}(F(x))=F(\operatorname{Im}(x))$ for any $x$ in $B$.

(3) If $\left\{x_{\alpha}\right\}_{\alpha \in A}$ is an arbitrary family of real elements of $B$ bounded above by some element $z$ of $B$, then $\bigvee_{\alpha} F\left(x_{\alpha}\right)$ exists and is equal to $F\left(\bigvee_{\alpha} x_{\alpha}\right)$.

(4) $F(|x|)=|F(x)|$ for any $x$ in $B$.

Proof. Assertions (1) and (2) are trivial. To show (3) first let $x$ be a real element of $B$. Then $x^{+} \geqq x, 0$, so $F\left(x^{+}\right) \geqq F(x), 0$, and so $F\left(x^{+}\right)$ $\geqq F(x)^{+}$. Similarly, $F\left(x^{-}\right) \geqq F(x)^{-}$. Thus 


$$
F(|x|)=F\left(x^{+}+x^{-}\right)=F\left(x^{+}\right)+F\left(x^{-}\right) \geqq F(x)^{+}+F(x)^{-}=|F(x)| .
$$

But, using axiom (I),

$$
\|F(|x|)-|F(x)|\|=\|F(|x|)\|-\||F(x)|\|=\|x\|-\|x\|=0,
$$

so that $F(|x|)=|F(x)|$. Then, in view of the relation

$$
x \bigvee y=\frac{1}{2}(x+y+|x-y|),
$$

it follows that $F(x \bigvee y)=F(x) \bigvee F(y)$ for any $x, y$ in $B$.

Then by induction, it follows that for any finite number of real elements $x_{1}, \ldots, x_{n}$ of $B$

$$
F\left(\bigvee_{i=1}^{n} x_{i}\right)=\bigvee_{i=1}^{n} F\left(x_{i}\right)
$$

Finally, if $\left\{x_{\alpha}\right\}_{\alpha \in A}$ is an arbitrary family of real elements of $B$ bounded above by some element $z$ of $B$, then $F\left(x_{\alpha}\right)$ is bounded above by $F(z)$ and so, in view of the lattice completeness of both $B$ and $C, x=\bigvee_{\alpha} x_{\alpha}$ and $w=\bigvee_{\alpha} F\left(x_{\alpha}\right)$ both exist. But

$$
\left\|x-\bigvee_{\alpha \in E} x_{\alpha}\right\| \rightarrow 0 \text { and }\left\|w-\bigvee_{\alpha \in E} F\left(x_{\alpha}\right)\right\| \rightarrow 0
$$

as $E$ ranges over the net of finite subsets of $A\left[2\right.$, p. 248]. Since $F\left(\bigvee_{\alpha \in E} x_{\alpha}\right)$ $=\bigvee_{\alpha \in E} F\left(x_{\alpha}\right)$,

$$
\|F(x)-w\| \leqq\left\|F(x)-F\left(\bigvee_{\alpha \in E} x_{\alpha}\right)\right\|+\left\|\bigvee_{\alpha \in E} F\left(x_{\alpha}\right)-w\right\| \rightarrow 0,
$$

so that we must have $F\left(\bigvee_{\alpha} x_{\alpha}\right)=\bigvee_{\alpha} F\left(x_{\alpha}\right)$. The proof of (4) is now immediate

$$
|F(x)|=\bigvee_{\theta} \operatorname{Re}\left(e^{i \theta} F(x)\right)=F\left(\bigvee_{\theta} \operatorname{Re}\left(e^{i \theta} x\right)\right)=F(|x|) .
$$

2. $L$-inducing functionals. Let $B$ be a complex Banach space, and let $f$ be a nonzero (continuous) linear functional on $B$. Define

$$
P=P(f)=\{x: x \in B, \text { and } f(x)=\|f\|\|x\|\} .
$$

2.1. Proposition. $P$ is a norm closed cone, and as such induces a partial order on $B$. With this order $B$ satisfies axiom (I).

Proof. If $x \in P$ and $\alpha$ is a positive real number, then

$$
f(\alpha x)=\alpha f(x)=\alpha\|f\|\|x\|=\|f\|\|\alpha x\|
$$

so that $\alpha x \in P$.

If $x, y \in P$ then

$$
\begin{aligned}
\|f\|\|x+y\| & \leqq\|f\|\|x\|+\|f\|\|y\|=f(x)+f(y) \\
& =f(x+y) \leqq\|f\|\|x+y\| .
\end{aligned}
$$


Thus $x+y \approx P$.

I $x,-x+-P$, then

$$
\|f\|\|x\|=\|f\|\|-x\|=f(-x)=-f(x)=-\|f\|\|x\|,
$$

so that $\|x\|=0$ since $\|f\| \neq 0$, and $x=0$.

It is clear from the definition that $P$ is closed. The order induced by $P$ is defined, of course, by $x \geqq y$ if and only if $x-y \in P$.

Finally, if $x \geqq 0, y \geqq 0$, so that $x, y, x+y \in P$, then

$$
\|f\|\|x+y\|=f(x+y)=f(x)+f(y)=\|f\|(\|x\|+\|y\|) .
$$

Since $\|f\| \neq 0,\|x+y\|=\|x\|+\|y\|$.

We will call the order described above the order on $B$ induced by $f$.

For reasons which will soon be apparent, we shall be most interested in linear functionals of the following type:

2.2. Definition. If $B$ is a Banach space, and $f$ is a linear functional on $B$, then $f$ will be said to be $L$-inducing if the order it induces on $B$ also satisfies axioms (IV)-(VII).

We now summarize some obvious facts concerning Banach spaces which possess at least one $L$-inducing linear functional.

2.3. Proposition. If $B$ is a Banach space which possesses an L-inducing linear functional, $f$, then there exists a locally compact Hausdorff space, $X$, and a positive regular Borel measure, $m$, on $X$, such that $B$ is isometrically linear isomorphic and, under the order induced by $f$, order isomorphic to $L(X, m)$. The dual of $B$ is then represented by $L^{\infty}(X, m)$, and under this representation $f$ is represented by the constant function $\|f\|$ on $X$.

Proof. By the definition of an $L$-inducing linear functional, $B$ with the order induced by $f$ becomes an abstract complex $L$-space, and so our extension of Kakutani's theorem applies. The only new fact here is that $f$ is represented by $\|f\|$. But if the representation of $B$ is denoted as before by $K$, then we have for $x \geqq 0$,

$$
\langle x, f\rangle=\|f\|\|x\|=\|f\|\|K(x)\|=\int(K(x))(s)\|f\| d m(s),
$$

so the result is true if $x \geqq 0$, and we can extend by linearity to the rest of $B$.

That a Banach space which has one $L$-inducing linear functional has, in fact, a very large number of $L$-inducing linear functionals follows from the following lemma and proposition which characterize the $L$-inducing linear functionals of concrete $L$-spaces.

2.4. Lemma. Let $L(X, m)$ be a complex concrete $L$-space, let $x$ be in $L(X, m)$, and let $f$ be in $L^{\infty}(X, m)$. Then $x$ in in $P(f)$ if and only if $x f=|x|\|f\|$.

Proof. Suppose that $x$ is in $P(f)$. Then $\langle x, f\rangle=\|f\|\|x\|$, which can be written as: 


$$
\int(\|f\||x(s)|-x(s) f(s)) d m(s)=0 \text {. }
$$

Then the real part of this integral is also 0 , i.e.,

$$
\int(\|f\||x(s)|-\operatorname{Re}(x(s) f(s))) d m(s)=0 .
$$

But $\operatorname{Re}(x(s) f(s)) \leqq|x(s)||f(s)| \leqq|x(s)|\|f\|$ a.e., and so, for the above integral to be 0 , we must have

$$
\operatorname{Re}(x(s) f(s))=|x(s)||f(s)|=|x(s)|\|f\| \text { a.e. }
$$

It follows that $\operatorname{Im}(x(s) f(s))=0$ a.e. and so

$$
x(s) f(x)=|x(s)|\|f\| \text { a.e. }
$$

The converse is clear.

2.5. Proposition. Let $L(X, m)$ be a complex concrete $L$-space, and let $f$ be in $L^{\infty}(X, m)$, with $f \neq 0$. Then $f$ is L-inducing if and only if $|f|=\|f\|$.

Proof. Suppose that $|f|=\|f\|$. Then $g=\|f\| / f$ is a well-defined element of $L^{\infty}(X, m),|g|=1$, and $\|g\|_{\infty}=1$. Then it is clear from the above lemma that $x$ is in $P(f)$ if and only if $x=y g$, where $y$ is in $L(X, m)$ and $y \geqq 0$. We now use a device which we will also use several times later. Consider the map, $J$, of $L(X, m)$ into itself defined by $J(x)=x g . J$ is clearly linear and bijective. It is an isometry since $\|J(x)\|=\int|g x|=\int|x|=\|x\|$. Finally, it is clear that $J(x)$ is in $P(f)$ if and only if $x \geqq 0$, and so $J$ is an order isomorphism of $L(X, m)$, with its ordinary order, onto $L(X, m)$ with the order induced by $f$. Since $L(X, m)$ with its ordinary order satisfies axioms (I), (III)-(VII) it follows that $L(X, m)$ with the order induced by $f$ does also.

Conversely, suppose that $f$ is $L$-inducing. Of course $|f| \leqq\|f\|$. But suppose that $|f| \neq\|f\|$. Then there is some set, $E$, of strictly positive finite measure, and an $e>0$, such that $|f(s)| \leqq\|f\|-e$ a.e. on $E$. Then by Lemma 2.4, if $x$ is in $P(f)$, then $x f=|x|\|f\|$, and so $|x(s)|\|f\|$ $=|x(s)| f(s)|\leqq| x(s)||(\|f\|-e)$ a.e. on $E$. But this can only be so if $|x(s)|=0$ a.e. on $E$. But $c$, the characteristic function of $E$, is an element of $L(X, m)$ which is not equal to 0 a.e. on $E$. Then it is clear that $c$ cannot be of the form $c=x-y+i(z-w)$ where $x, y, z, w$, are in $P(f)$. Thus $f$ is not $L$-inducing.

2.6. Corollary. If $f$ is L-inducing, then $x$ is in $P(f)$ if and only if $x=|x|\|f\| / f$.

2.7. Corollary. If $|f|=1$, then $f$ is L-inducing, and $x$ is in $P(f)$ if and only if $x=|x| \bar{f}$. 
Since it may appear that up to now we have hardly discussed measure snaris may be appropriate at this juncture to point out that this is not the case. If $X$ is a locally compact Hausdorff space and $M(X)$ is the Banach space of all finite complex regular Borel measures on $X$, then under its natural order $M(X)$ satisfies axioms (I) and (III) -(VII), and so is an abstract complex $L$-space, which, of course, leads to the slightly strange conclusion that $M(X)$ is representable as $L(Y, m)$ where $Y$ is some other locally compact Hausdorff space with Borel measure $m$. The existence of this representation is the key for relating much of the sequel to measure algebras.

For the purposes of motivation in the next section it will be useful to have the following result:

2.8. Proposition. Let $f$ be a bounded measurable function on $X$, so that $f$ represents a linear functional on $M(X)$. Then $f$ is L-inducing if and only if $|f(s)|=\|f\|$ for all $s$ in $X$.

Proof. It is clear how to modify the proof of Proposition 2.5 to obtain this result.

3. Group algebras and measure algebras. Let $G$ be a locally compact Abelian (l.c.a.) group. Let $L(G)$ and $M(G)$ be the group algebra and measure algebra respectively of $G$. Then if $f$ is any (continuous) character of $G$, $f$ has the property that $1=|f(s)|=\|f\|_{\infty}$ for all $s$ in $G$, and so by Propositions 2.5 and 2.8, the corresponding multiplicative linear functional (m.l.f.) is $L$-inducing on both $L(G)$ and $M(G)$. Thus both $L(G)$ and $M(G)$ have a separating family of $L$-inducing m.l.f. Our main result roughly stated, says that this property characterizes these algebras. We will need to strengthen slightly the definition of $L$-inducing linear functional so that it relates nicely to the multiplication in Banach algebras. This will be done in this section. We will begin by considering the relation between a m.l.f. of an arbitrary Banach algebra and the order induced by this m.l.f.

But first a remark on our notation is in order. Throughout the sequel, $A$ will represent a complex commutative Banach algebra, with the product of two elements, $x, y$, of $A$ written as $x * y$. $A$ is never assumed to have an identity element or any kind of approximate identity. In the course of later discussion, elements, $x$, of $A$ will be represented as functions on a set $X$, in which case, if $f$ is any other function on $X$, then $x f$ will denote the pointwise product of $x$ with $f$. This is why we use a separate symbol for the multiplication in $A$. Elements, $x$, of $A$ may also be represented as measures on a locally compact Hausdorff space $X$, in which case $x f$ will denote the measure on $X$ defined by $(x f)(c)=\int c(s) f(s) d x(s)$ for all continuous functions, $c$, of compact support in $X$.

As implied above, the fact that a linear functional on a Banach algebra, 
$A$, is multiplicative can lead to relations between the order induced by the functional and the multiplication in $A$. To begin with:

3.1. Proposition. Let $A$ be a commutative Banach algebra, and let $f$ be a m.l.f. of $A$, with $\|f\|=1$. Then if $x$ and $y$ are positive with respect to the order induced by $f$, then so is $x * y$. If $x$ and $y$ are real, so is $x * y$.

Proof. If $x$ and $y$ are in $P(f)$, then

$$
\|x\|\|y\|=f(x) f(y)=f(x * y) \leqq\|x * y\| \leqq\|x\|\|y\| .
$$

In particular, the situation described in 3.1 holds in $L(G)$ and $M(G)$ when $f$ is a character of $G$.

Another interesting relation between the multiplication and the order induced by a m.l.f. occurs in group and measure algebras:

3.2. Proposition. Let $G$ be a l.c.a. group, and let $f$ be the m.l.f. on $L(G)$, or $M(G)$, represented by some character of $G$. Then for any $x, y$

$$
|x * y| \leqq|x| *|y|
$$

where the absolute value, || , is with respect to the order induced by $f$.

Proof. To prove this for both $L(G)$ and $M(G)$ simultaneously, we view the elements of $L(G)$ as measures. We first show that relation (3.3) holds with respect to the ordinary order in $L(G)$ and $M(G)$. Let us recall the definitions which we will use [15]. If $x \in M(G)$ and $E$ is any Borel subset of $G$, then $|x|$ can be defined by

$$
|x|(E)=\sup \left\{\sum\left|x\left(E_{i}\right)\right|: \oplus E_{i}=E\right\},
$$

where by $\oplus E_{i}=E$ we mean that $E$ is the disjoint union of the finite collection $\left\{E_{i}\right\}$ of Borel subsets. If $x, y \in M(G)$ and $E$ is any Borel subset of $G$, then $x * y$ is defined by

$$
(x * y)(E)=\int x(E-s) d y(s) .
$$

Thus if $\oplus E_{i}=E$, then

$$
\begin{aligned}
\sum\left|(x * y)\left(E_{i}\right)\right| & =\sum\left|\int x\left(E_{i}-s\right) d y(s)\right| \leqq \sum \int\left|x\left(E_{i}-s\right)\right| d|y|(s) \\
& \leqq \sum \int|x|\left(E_{i}-s\right) d|y|(s)=(|x| *|y|)(E)
\end{aligned}
$$

Since $E$ is an arbitrary Borel subset, it is clear that

$$
|x * y| \leqq|x| *|y| \text {. }
$$

The key to the general case is the following easily verified relation, an 
analog ?e of which will play an important role later in an abstract setting .i. For $x, y \in M(G)$ and for any character, $f$, of $G$

$$
(x \bar{f}) *(y \bar{f})=(x * y) \bar{f} \text {. }
$$

We now use the device used in the proof of Proposition 2.5. Let $J$ be the map of $L(G)$ or $M(G)$ into itself defined by $J(x)=x \bar{f}$. It is clear, as before, that $J$ is an isometric linear and order isomorphism of $L(G)$ (resp. $M(G)$ ), with its ordinary order, onto $L(G)$ (resp. $M(G)$ ) with the order induced by $f$. Further, by (3.4),

$$
J(x * y)=(x * y) \bar{f}=(x \bar{f}) *(y \bar{f})=(J(x)) *(J(y)) .
$$

Thus $J$ is also an algebra isomorphism, and the conclusion follows.

These observations lead to the following definition:

3.5. Definition. Let $A$ be a commutative Banach algebra, and let $f$ be a nonzero m.l.f. on $A$. Then $f$ will be said to be $L^{\prime}$-inducing if, as a linear functional on $A$, it is $L$-inducing, and if, further, it satisfies the following additional axioms:

(VIII) $\|f\|=1$ (so that 3.1 applies).

(IX) For all $x, y$ in $A,|x * y| \leqq|x| *|y|$ (where, of course, || is taken with respect to the order induced by $f$ ).

We do not know whether axioms (VIII) and (IX) are independent of axioms (IV)-(VII), though we suspect that they are not. In the real case axiom (IX) does follow trivially from axioms (IV)-(VII) and (VIII).

It is clear from 3.2 that both $L(G)$ and $M(G)$ have a separating family of $L^{\prime}$-inducing m.l.f.

4. Proof of Theorem B. Now that we have given the definition of an $L^{\prime}$ inducing m.l.f. we are in a position to prove Theorem $B$, which we restate here in slightly more detail.

4.1.1. Theorem B. Let $A$ be a complex commutative Banach algebra, and let $D$ be the collection of all $L^{\prime}$-inducing m.l.f. of $A$. Suppose that

(1) $D$ is a separating family of linear functionals for $A$.

Then there exists a compact Abelian group, $B$, such that $A$ is isometrically algebra isomorphic to a (closed) subalgebra of $M(B)$. If $f$ is any fixed element of $D$, then this isomorphism can be chosen to be also an order isomorphism of $A$, with order induced by $f$, into $M(B)$ with its ordinary order.

Suppose in addition that

(2) $D$ is locally compact in the $w^{*}$ topology.

Then there exists a locally compact Abelian group, $G$, whose dual group is homeomorphic to $D$, and whose Bohr compactification is $B$, such that $A$ is isometrically algebra isomorphic to a (closed) subalgebra of $M(G)$. As before, if $f$ is any fixed element of $D$, then this isomorphism can be chosen to be also an order 
isomorphism of $A$, with order induced by $f$, into $M(G)$ with its ordinary order.

Proof. Throughout the proof $f$ will be an arbitrarily chosen fixed element of $D$, and is the $f$ referred to in the statement of the theorem.

Since $f$ is $L^{\prime}$-inducing, we can apply Proposition 2.3 to obtain a locally compact Hausdorff space, $X$, a positive regular Borel measure, $m$, on $X$, and an isometric linear isomorphism of $A$ with $L(X, m)$. This isomorphism is also an order isomorphism of $A$, with order induced by $f$, onto $L(X, m)$ with its natural order. Throughout $\$ 4, X, m$, and this isomorphism will remain fixed, and whenever convenient we will identify $A$ with $L(X, m)$.

The dual of $A$ can then be identified with $L^{\infty}(X, m)$, and under this identification, $f$ is represented by the constant function 1 on $X$. As a result, since $f$ is a m.l.f. of $A$,

$$
\int(x * y) d m=\left(\int x d m\right)\left(\int y d m\right) \quad(x, y \in A) .
$$

Also, $D$ can be identified with a subset of $L^{\infty}(X, m)$, and under this identification, if $g$ is in $D$, then, by $2.5,|g|=1$.

All statements concerning order will be with respect to the natural order on $L(X, m)$, i.e., the order induced by $f$, unless the contrary is explicitly stated. Integration will always be with respect to $m$ unless the contrary is explicitly stated, e.g. we will usually write (4.1.2) in the form

$$
\int(x * y)=\int x \int y \text {. }
$$

4.2. The "dual" group. We now show that $D$ under pointwise multiplication is an Abelian group, and further, that if hypothesis (2) is satisfied, then $D$, with the $w^{*}$ topology, is a locally compact Abelian group. This group will turn out to be the dual of the group $G$ of the second half of the theorem.

4.2.1. Lemma. If $g \in D$ then $(x \bar{g}) *(y \bar{g})=(x * y) \bar{g}$ for all $x, y \in A$ (compare with (3.4)).

Proof. Suppose first that $x$ and $y$ are positive. From 2.7 it follows that $x \bar{g}$ and $y \bar{g}$ are in $P(g)$, and so from $3.1,(x \bar{g}) *(y \bar{g})$ is in $P(g)$, and $x * y \geqq 0$. Then again, by $2.7,(x * y) \bar{g}$ is in $P(g)$, and $(x \bar{g}) *(y \bar{g})=|(x \bar{g}) *(y \bar{g})| \bar{g}$. Thus by axiom (IX), and from the fact that $\bar{g} g=1$,

$$
\begin{aligned}
((x \bar{g}) *(y \bar{g})) g & =|(x \bar{g}) *(y \bar{g})| \bar{g} g=|(x \bar{g}) *(y \bar{g})| \\
& \leqq|x \bar{g}| *|y \bar{g}|=|x| *|y|=x * y .
\end{aligned}
$$

Thus 


$$
0 \leqq((\bar{g}) *(y \bar{g})) g \leqq x * y .
$$

But since $g$ is a m.l.f.

$$
\int((x \bar{g}) *(y \bar{g})) g=\int(x \bar{g}) g \int(y \bar{g}) g=\int x \int y=\int x * y .
$$

In view of this, (4.2.2) can hold only if

$$
((x \bar{g}) *(y \bar{g})) g=x * y \text {. }
$$

For general $x$ and $y$ we extend the above result by linearity.

We now prove a partial converse of 4.2.1.

4.2.3. Lemma. Let $g \in L^{\infty}(X, m)$ and suppose that

1. There exists a real number $k>0$ such that $|g| \geqq k$,

2. $(x \bar{g}) *(y \bar{g})=(x * y) \bar{g}$ for all $x, y \in A$.

Then $g$ is an $L^{\prime}$-inducing m.l.f.

Proof. We first note that $(x * y)^{-}=\bar{x} * \bar{y}$ for any $x, y \in A$ (where ${ }^{-}$denotes complex conjugation). For if $x=x^{\prime}+i x^{\prime \prime}, y=y^{\prime}+i y^{\prime \prime}$ where $x^{\prime}, x^{\prime \prime}$, $y^{\prime}, y^{\prime \prime}$ are real, then

$$
\bar{x} * \bar{y}=\left(x^{\prime}-i x^{\prime \prime}\right) *\left(y^{\prime}-i y^{\prime \prime}\right)=\left(x^{\prime} * y^{\prime}-x^{\prime \prime} * y^{\prime \prime}\right)-i\left(x^{\prime \prime} * y^{\prime}+x^{\prime} * y^{\prime \prime}\right),
$$

which by 3.1 is seen to be $(x * y)^{-}$. Then, for $x, y \in A$,

$$
(x * y) g=((\bar{x} * \bar{y}) \bar{g})^{-}=((\bar{x} \bar{g}) *(\bar{y} \bar{g}))^{-}=(x g) *(y g) .
$$

Now let $h=g^{-1}$. Since $|g| \geqq k$, we have $|h| \leqq 1 / k$ so that $h \in L^{\infty}(X, m)$. Further, since $g h=1$, we have

$$
(x h) *(y h)=((x h) *(y h)) g h=((x h g) *(y h g)) h=(x * y) h .
$$

Then both $g$ and $h$ are m.l.f. of $A$, for if $x, y \in A$, then

$$
\int(x * y) g=\int(x g) *(y g)=\int x g \int y g
$$

and similarly for $h$. But any m.l.f. of a Banach algebra has norm $\leqq 1$ $[12$, p. 52$]$, so $\|g\| \leqq 1$ and $\|h\| \leqq 1$. Thus $|g| \leqq 1$ and $\left|g^{-1}\right|=|h| \leqq 1$, so that $|g|=1$. Thus, by $2.5, g$ is an $L$-inducing linear functional, and $g$ clearly satisfies axiom (VIII).

It remains to show that $g$ satisfies axiom (IX). We use the device used to prove 2.5 and 3.2. Let $J$ be the map of $A$ onto itself defined by $J(x)$ $=x \bar{g}$. Then, as before, $J$ is an isometric algebra and order isomorphism of $A$ with its ordinary order onto $A$ with the order induced by $g$. Since the ordinary order satisfies axiom (IX), so does the order induced by $g$.

4.2.4. We now show that $D$ is a group under pointwise multiplication. If $g, h \in D$, then clearly $|g h|=1$. Further, by (4.2.1), for all $x, y \in A$, 


$$
(x \bar{g} \bar{h}) *(y \bar{g} \bar{h})=((x \bar{g}) *(y \bar{g})) \bar{h}=(x * y) \bar{g} \bar{h} .
$$

Thus by $4.2 .3, g h$ is in $D$, and so $D$ is closed under pointwise multiplication. Next, the constant function 1 , which is $f$, is in $D$, and so $D$ contains an identity for pointwise multiplication. Finally, if $g \in D$, and if we let $h=g^{-1}$, then $|h|=1$, and by the arguments in the proof of 4.2.3, $(x \bar{h}) *(y \bar{h})$ $=(x * y) \bar{h}$ for all $x, y \in A$, and so by $4.2 .3, h=g^{-1}$ is in $D$.

4.2.5. We now show that $D$ is a topological group in the $w^{*}$ topology. That this can be done before assuming that $D$ is locally compact in the $w^{*}$ topology, as we had done in our original proof, was pointed out to us by E. G. Effros. The proof which he indicated is as follows: Consider $L^{\infty}(X, m)$ as an algebra of bounded operators on $L^{2}(X, m)$ by pointwise multiplication in the usual way. Then $D$ will be a subgroup of the group of unitary operators on $L^{2}(X, m)$. Also, the $w^{*}$ topology on $D$ will coincide with the weak operator topology on $D$, as the following relations make clear:

4.2.6. (1) If $u, v \in L^{2}(X, m)$ and $f \in D$, then $u \bar{v} \in L(X, m)$ and

$$
\langle f u, v\rangle=\int f u \bar{v}=\langle u \bar{v}, f\rangle .
$$

(2) Conversely, if $x \in L(X, m)$ let $u=|x|^{1 / 2}, v=\bar{x} / u$. Then $u, v \in L^{2}(X, m)$, $x=u \bar{v}$, and

$$
\langle x, f\rangle=\int f u \bar{v}=\langle f u, v\rangle .
$$

Furthermore, on the group of unitary operators, and so on the subgroup $D$, the weak operator topology coincides with the strong operator topology, as is seen by the fact that for $u \in L^{2}(X, m)$ and for $f, f_{0} \in D$

$$
\left\|\left(f-f_{0}\right) u\right\|_{2}^{2}=\left\langle\left(f_{0}-f\right) u, f_{0} u\right\rangle+\left\langle f_{0} u,\left(f-f_{0}\right) u\right\rangle
$$

since

$$
\langle f u, f u\rangle=\langle u, u\rangle=\left\langle f_{0} u, f_{0} u\right\rangle .
$$

But it is easy to check that multiplication of unitary operators is jointly continuous in the strong operator topology, and that taking inverses is continuous in the weak operator topology. Thus $D$ is a topological group in the strong operator and weak operator topology, and so in the $w^{*}$ topology.

4.2.7. It is now clear that if hypothesis (2) is satisfied, then $D$ with the $w^{*}$ topology is a locally compact Abelian group.

4.3. The "Bohr compactification". Birtel [4] considers an arbitrary commutative semisimple Banach algebra, $A$, with dual as a Banach space, $A^{*}$. He lets $A^{\prime}$ be the norm-closed subspace of $A^{*}$ spanned by the m.l.f. of $A$, and lets $A^{\prime \prime}$ be the Banach space dual of $A^{\prime}$. He then shows, in analogy with the usual double dual of an algebra, that $A^{\prime \prime}$ is a commutative semi- 
simple Banach algebra with a multiplication induced by the multiplication ic atural map of $A$ into $A^{\prime \prime}$ is a continuous algebra injection, but he is not able to conclude that it is an isometry.

We will follow this program, but instead of taking $A^{\prime}$ to be the normclosed subspace spanned by all the m.l.f. of $A$, we will take $A^{\prime}$ to be the norm-closed subspace spanned by $D$. Because of our added hypotheses on $A$, we will be able to obtain much more information than could be obtained in Birtel's more general setting.

To make the above precise, consider the linear span of $D$ in $L^{\infty}(X, m)$. Since $D$ is closed under pointwise multiplication, this span is already a subalgebra of $L^{\infty}(X, m)$. Since it is also clear that the span of $D$ is closed under complex conjugation, the norm-closure of the span of $D$ will be a sub- $C^{*}$-algebra of $L^{\infty}(X, m)$, with unit $f=1$. We will denote this sub- $C^{*}$ algebra by $P$, instead of $A^{\prime}$, since it should be thought of as consisting of the almost periodic functions in the present situation. Let $B$ be the maximal ideal space of $P$. Then $B$ is a compact Hausdorff space, and should be thought of as the Bohr compactification of the group on which we wish to place $A$. $P$ can, of course, be identified with $C(B)$, the continuous complexvalued functions on $B$, and we will almost always make this identification. The analogue of Birtel's $A^{\prime \prime}$ is then just the dual of $C(B)$, that is, the space $M(B)$ of all regular Borel measures on $B$.

4.4. The isometric embedding. There is, of course, a natural (isometric) embedding of $A$ (as $L(X, m)$ ) into the dual of $L^{\infty}(X, m)$. Viewing $A$ as so embedded, the restriction of $A$ to $P$ is a norm nonincreasing linear map of $A$ into the dual of $P$, that is, into $M(B)$. We denote this map by $K$, so that for $x$ in $A$ and $h$ in $C(B), K(x)(h)=\langle x, h\rangle$. Since by hypothesis (1), $D$, and hence $P$, is a separating family of linear functionals on $A$, it is clear that $K$ is injective. We now show that $K$ is in fact an isometry.

Consider $P$ as a closed subspace of $L^{\infty}(X, m)$. As above, hypothesis (1) implies that the annihilator of $P$ in $A$ is just $\{0\}$. This means that $P$ is dense in $L^{\infty}(X, m)$ in the $w^{*}$ topology. Now consider $L^{\infty}(X, m)$ and its sub- $C^{*}$-algebra, $P$, as bounded operators on $L^{2}(X, m)$ as we did in 4.2.5. Then [7, p. 117] $L^{\infty}(X, m)$ is a von Neumann algebra, and by the relations 4.2.6 the weak operator topology on $L^{\infty}(X, m)$ is the same as the $w^{*}$ topology on $L^{\infty}(X, m)$. Thus $P$ is dense in $L^{\infty}(X, m)$ in the weak operator topology and so also in the strong operator topology. We can thus apply the Kaplansky density theorem $[7$, p. 46] (we are indebted to E. G. Effros for bringing this theorem to our attention) to assert that the unit ball of $P$ is dense in the unit ball of $L^{\infty}(X, m)$ in the strong operator, hence weak operator, hence $w^{*}$ topology.

This is a sufficient condition for the map $K$ of $A$ into the dual of $P$ to be an isometry, for, given $x$ in $A$, the norm of $x$ as an element of $A$ is 


$$
\sup \left\{|\langle x, g\rangle|: g \text { is in the unit ball of } L^{\infty}(X, m)\right\} .
$$

But $|\langle x, \cdot\rangle|$ is a continuous function on the unit ball of $L^{\infty}(X, m)$ with the $w^{*}$ topology, and so, since the unit ball of $P$ is dense in the unit ball of $L^{\infty}(X, m)$ with the $w^{*}$ topology, (4.4.1) is equal to

$$
\sup \{|\langle x, g\rangle|: g \text { is in the unit ball of } P\} \text {. }
$$

But this is just the norm of $x$ as an element of the dual of $P$. Thus $K$ embeds $A$ isometrically in $M(B)$.

4.5. Preservation of order. We now point out that $K$ is order preserving. This follows immediately from the fact that $K$ is isometric, and the fact that $f$, the constant 1 , induces the ordinary order in $M(B)$, as well as inducing the order in $A$. Thus $\langle x, f\rangle=\|x\|$ if and only if $K(x)(f)=\|x\|$ $=\|K(x)\|$, and so $x$ is in $P(f)$ if and only if $K(x)$ is in $P(f)$. Thus $x \geqq 0$ if and only if $K(x) \geqq 0$. We remark that we are thus in a position to apply Proposition 1.8 .

4.6. Multiplication in $M(B)$. In analogy with Birtel's construction, we now show that the multiplication in $A$ induces a Banach algebra multiplication in all of $M(B)$. We will denote elements of $M(B)$ by $F, G, H$ as well as by the $K(x)$ which come from $A$.

The definition of the multiplication is in three steps, each of which consists of defining certain operations on the linear span of $D$, and then extending these operations by uniform continuity to all of $C(B)$. Throughout, a general element of the span of $D$ will be represented by the finite sum $\sum a_{i} g_{i}$, where the $g_{i}$ are in $D$, and the $a_{i}$ are scalars.

4.6.1. For $x$ in $A$ and $\sum a_{i} g_{i}$ in the span of $D$ define an element, $x \otimes\left(\sum a_{i} g_{i}\right)$, of $C(B)$ by

$$
x \otimes\left(\sum a_{i} g_{i}\right)=\sum a_{i}\left\langle x, g_{i}\right\rangle g_{i}
$$

(that this product is independent of the representative $\sum a_{i} g_{i}$ is established by (4.6.2)). Then for $y$ in $A$

$$
\begin{aligned}
\left|\left\langle y, x \otimes\left(\sum a_{i} g_{i}\right)\right\rangle\right| & =\left|\left\langle y, \sum a_{i}\left\langle x, g_{i}\right\rangle g_{i}\right\rangle\right| \\
& =\left|\sum a_{i}\left\langle x, g_{i}\right\rangle\left\langle y, g_{i}\right\rangle\right|=\left|\sum a_{i}\left\langle x * y, g_{i}\right\rangle\right| \\
& =\left|\left\langle x * y, \sum a_{i} g_{i}\right\rangle\right| \leqq\|x * y\|\left\|\sum a_{i} g_{i}\right\| \\
& \leqq\|x\|\|y\|\left\|\sum a_{i} g_{i}\right\| .
\end{aligned}
$$

Thus

$$
\left\|x \otimes\left(\sum a_{i} g_{i}\right)\right\| \leqq\|x\|\left\|\sum a_{i} g_{i}\right\| .
$$

Then for any fixed $x$ in $A$ the linear map

$$
\left.\left(\sum a_{i} g_{i}\right) \rightarrow x \otimes i \sum a_{i} g_{i}\right)
$$


is unif mly continuous on a dense subset of $C(B)$, and so extends uniquely to a linear map, $h \rightarrow x \otimes h$, of $C(B)$ into itself satisfying $\|x \otimes h\| \leqq\|x\|$ $\|h\|$. For fixed $x, y$ in $A$ the maps

$$
h \rightarrow\langle y, x \otimes h\rangle \text { and } h \rightarrow\langle x * y, h\rangle
$$

are continuous, and as in (4.6.2), they agree on the span of $D$. Thus for all $x, y$ in $A$ and $h$ in $C(B)$

$$
\langle y, x \otimes h\rangle=\langle x * y, h\rangle .
$$

Clearly $x \otimes h$ is linear in $x$.

4.6.3. If $F$ is in $M(B)$, let $\langle F, h\rangle=F(h)$ for any $h$ in $C(B)$. Thus for all $x$ in $A$ and $h$ in $C(B)$

$$
\langle K(x), h\rangle=\langle x, h\rangle .
$$

Then for $F$ in $M(B)$, and for $\sum a_{i} g_{i}$ in the span of $D$, define an element, $F \otimes\left(\sum a_{i} g_{i}\right)$, of $C(B)$ by

$$
F \otimes\left(\sum a_{i} g_{i}\right)=\sum a_{i}\left\langle F, g_{i}\right\rangle g_{i} .
$$

As in (4.6.2) it can be checked that

$$
\left\|F \otimes\left(\sum a_{i} g_{i}\right)\right\| \leqq\|F\|\left\|\sum a_{i} g_{i}\right\| .
$$

Thus for any fixed $F$ the linear map

$$
\left(\sum a_{i} g_{i}\right) \rightarrow F \otimes\left(\sum a_{i} g_{i}\right)
$$

extends uniquely to a linear map, $h \rightarrow F \otimes h$, of $C(B)$ into itself satisfying $\|F \otimes h\| \leqq\|F\|\|h\|$, and as before, for all $x$ in $A, F$ in $M(B)$, and $h$ in $C(B)$

$$
\langle x, F \otimes h\rangle=\langle F, x \otimes h\rangle .
$$

Clearly $F \otimes h$ is linear in $F$.

4.6.4. Finally, for $F, G$ in $M(B)$ and for $\sum a_{i} g_{i}$, in the span of $D$, define $F * G$ by

$$
\begin{aligned}
\left\langle F * G, \sum a_{i} g_{i}\right\rangle & =\sum a_{i}\left\langle F, g_{i}\right\rangle\left\langle G, g_{i}\right\rangle \\
& =\left\langle F, \sum a_{i}\left\langle G, g_{i}\right\rangle g_{i}\right\rangle=\left\langle F, G \otimes\left(\sum a_{i} g_{i}\right)\right\rangle .
\end{aligned}
$$

Then, as above, $F * G$ extends uniquely to a bounded linear map

$$
h \rightarrow\langle F * G, h\rangle
$$

on $C(B)$ satisfying

$$
\|F * G\| \leqq\|F\|\|G\|,
$$

and so $F * G$ is in $M(B)$.

It is clear from the definition that $F * G$ and $G * F$ agree on the span of $D$ and so $F * G=G * F$ for all $F, G$ in $M(B)$. A routine check establishes 
the associativity and bilinearity of $*$. Thus under $*, M(B)$ becomes a commutative Banach algebra. As before, for all $F, G$ in $M(B)$ and $h$ in $C(B)$

$$
\langle F * G, h\rangle=\langle F, G \otimes h\rangle .
$$

That $K$ is an algebra embedding of $A$ into $M(B)$ is easily checked by considering the span of $D$ as above.

4.6.6. It will be important later to know that $*$ is separately continuous in the $w^{*}$ topology on $M(B)$ as dual of $C(B)$. To see this let $F_{\alpha}$ be a net of elements in $M(B)$, and let $F_{\alpha} \rightarrow F$ in the $w^{*}$ topology, where $F \in M(B)$. Then for fixed $G$ in $M(B)$, and for any $h$ in $C(B), G \otimes h$ is in $C(B)$, and so

$$
\left\langle F_{\alpha} * G, h\right\rangle=\left\langle F_{a}, G \otimes h\right\rangle \rightarrow\langle F, G \otimes h\rangle=\langle F * G, h\rangle,
$$

so that $F_{\alpha} * G \rightarrow F * G$ in the $w^{*}$ topology.

We remark here that, directly from the definition of $*$ in $M(B)$, each $g$ in $D$ is a m.l.f. of $M(B)$, that is,

$$
\langle F * G, g\rangle=\langle F, g\rangle\langle G, g\rangle \text { for all } F, G \text { in } M(B) .
$$

Further, it is clear that since the span of $D$ is dense in $C(B)$, the elements of $D$ separate the points of $M(B)$, so that $M(B)$ is a semisimple Banach algebra.

4.7. Multiplication in $B$. We now wish to use the multiplication in $M(B)$ to define a multiplication in $B$, using the natural identification of points of $B$ with positive unit point measures. To make this precise, if $r$ is in $B$, let $F(r)$ be the positive unit measure concentrated at $r$. Then if $r, s$ are in $B$, we will show that $F(r) * F(s)$ is also a positive unit point measure.

To begin with, if $g$ and $g^{\prime}$ are in $D$, then since $g g^{\prime}$ is also in $D$, it is easily checked that

$$
\left\langle F(r) * F(s), g g^{\prime}\right\rangle=\langle F(r) * F(s), g\rangle\left\langle F(r) * F(s), g^{\prime}\right\rangle .
$$

But any $F$ in $M(B)$ with the property that

$$
\left\langle F, g g^{\prime}\right\rangle=\langle F, g\rangle\left\langle F, g^{\prime}\right\rangle \quad \text { for all } g, g^{\prime} \text { in } D
$$

is a positive unit point measure, for, given elements $\sum a_{i} g_{i}, \sum b_{j} g_{j}^{\prime}$ in the span of $D$, it is easily checked that

$$
\left\langle F,\left(\sum a_{i} g_{i}\right)\left(\sum b_{j} g_{j}^{\prime}\right)\right\rangle=\left\langle F, \sum a_{i} g_{i}\right\rangle\left\langle F, \sum b_{j} g_{j}^{\prime}\right\rangle .
$$

And so by continuity, for any $h, h^{\prime} \in C(B)$,

$$
\left\langle F, h h^{\prime}\right\rangle=\langle F, h\rangle\left\langle F, h^{\prime}\right\rangle .
$$

Thus $F$ is a m.l.f. of $C(B)$, and so is a positive unit point measure. In particular $F(r) * F(s)$ is a positive unit point measure. 
This for any $r, s$ in $B$, there exists a unique point, $t$, of $B$ such that $f(r) * F(s)=F(t)$. We define a multiplication in $B$ by $r s=t$. The associativity and commutativity of $*$ in $M(B)$ imply that this multiplication in $B$ is also associative and commutative. Thus $B$ becomes a commutative semigroup.

4.8. $B$ is a topological group. We will now show that $B$, with the abovedefined multiplication, and with its compact Hausdorff topology, is a topological group.

4.8.1. We first show that the multiplication in $B$ is separately continuous in the topology of $B$. This follows from the separate continuity of $*$ in the $w^{*}$ topology on $M(B)$ (see 4.6.6), and the fact that the topology on $B$ is the same as the weak topology induced by the elements of $C(B)$.

Specifically, suppose $s_{\alpha}$ is a net of elements of $B$ which converges in the topology of $B$ to some element, $s$, of $B$, and let $r$ be any fixed element of $B$. Then for all $h$ in $C(B)$,

$$
\left\langle F\left(s_{\alpha}\right), h\right\rangle=h\left(s_{\alpha}\right) \rightarrow h(s)=\langle F(s), h\rangle,
$$

that is, $F\left(s_{\alpha}\right) \rightarrow F(s)$ in the $w^{*}$ topology. Then, by 4.6.6, $F(r) * F\left(s_{\alpha}\right)$ $\rightarrow F(r) * F(s)$ in the $w^{*}$ topology, so that $F\left(r s_{\alpha}\right) \rightarrow F(r s)$ in the $w^{*}$ topology. Thus for all $h$ in $C(B)$,

$$
h\left(r s_{\alpha}\right)=\left\langle F\left(r s_{\alpha}\right), h\right\rangle \rightarrow\langle F(r s), h\rangle=h(r s),
$$

and $r s_{\alpha} \rightarrow r s$ in the topology of $B$.

4.8.2. We now show that the cancellation law holds for the multiplication in $B$. It is easy to prove an even stronger result, namely, that if $r$ is in $B$ and if $G, H$ are in $M(B)$, and if $F(r) * G=F(r) * H$, then $G=H$. For, given the hypotheses, we have $F(r) *(G-H)=0$, and so for every $g$ in $D$,

$$
0=\langle F(r) *(G-H), g\rangle=\langle F(r), g\rangle\langle G-H, g\rangle .
$$

But every $g$ in $D$ has the property that $|g|=1$, and so $\langle F(r), g\rangle=g(r)$ $\neq 0$ for all $g$ in $D$. Thus $\langle G-H, g\rangle=0$ for all $g$ in $D$, and since $D$ is a separating family of linear functionals, we must have $G=H$.

Thus in particular, if $r s=r t$, so that $F(r) * F(s)=F(r s)=F(r t)$ $=F(r) * F(t)$, then $F(s)=F(t)$, and so $s=t$.

4.8.3. We are now in a position to use a theorem given in $[10, p .814]$ and attributed there to Iwasawa, which asserts that any semigroup with compact topology in which multiplication is separately continuous, and in which the cancellation law holds is actually a topological group. It follows that $B$ is a compact Abelian group.

4.9. The dual of $B$. We now show that the group $D$, with the discrete topology, is the dual group of $B$. Since for any $g$ in $D$ and any $r, s$ in $D$, 


$$
\begin{aligned}
g(r s) & =\langle F(r s), g\rangle=\langle F(r) * F(s), g\rangle \\
& =\langle F(r), g\rangle\langle F(s), g\rangle=g(r) g(s),
\end{aligned}
$$

and since $|g| \doteq 1$, it is clear that each $g$ in $D$ is a (continuous) character of $B$. The multiplication in $D$ is just the usual pointwise multiplication which one defines for characters, and so $D$ is a subgroup of the dual group of $B$. Furthermore, since $D$ is a separating family for $M(B)$, it is also one for $B$. Thus $D$ is the dual of $B$, for if it were a proper subgroup of the dual, then by the Pontrjagin duality theorem there would be a nontrivial subgroup of $B$ which would be in the kernel of every $g$ in $D[14$, p. 35].

4.10. * is convolution. We now show that * is convolution with respect to the multiplication in $B$. Given $F, G$ in $M(B)$, let $F \circ G$ be the actual convolution of $F$ and $G$ with respect to the multiplication in $B$. Then since the $g$ in $D$ are characters of $B$, for all $g$ in $D$

$$
\langle F \circ G, g\rangle=\langle F, g\rangle\langle G, g\rangle \text {. }
$$

Then

$$
\langle F \circ G-F * G, g\rangle=\langle F, g\rangle\langle G, g\rangle-\langle F, g\rangle\langle G, g\rangle=0
$$

for all $g$ in $D$, and so, since the elements of $D$ are separating, $F \circ G=F * G$, and $*$ is just convolution with respect to the multiplication in $B$.

This concludes the proof of the first part of the main theorem.

4.11. A on the dual of $D$. Suppose now that hypothesis (2) is satisfied, so that $D$ is locally compact in the $w^{*}$ topology, and so, by 4.2 .6 , is a locally compact Abelian group. Let $G$ be the dual of $D$. Since $B$ is the dual of $D$ with the discrete topology on $D$, it is clear that the Bohr compactification of $G$ is $B$. We wish to embed $A$ in $M(G)$.

If $D$ with the $w^{*}$ topology is discrete, than $G=B$, and we are done.

If $D$ is not discrete we proceed as follows. If $x$ is in $A$, denote by $\check{x}$ the restriction to $D$ of the Gelfand transform of $x$. Then, by the definition of the $w^{*}$ topology, $\check{x}$ is continuous on $D$. Further, from the relation

$$
\langle x, g\rangle=\langle K(x), g\rangle \text { for all } g \text { in } D,
$$

it is clear that $\check{x}$ is also the inverse Fourier-Stieltjes transform of $K(x)$. Then the Fourier-Stieltjes transform, $\hat{x}$, of $K(x)$ is $\hat{x}(g)=\check{x}\left(g^{-1}\right)=\check{x}(\bar{g})$, and so is itself continuous and hence measurable with respect to Haar measure on $D$. But the Fourier-Stieltjes transform, $\hat{F}$, of any element, $F$, of $M(B)$ satisfies

$$
\left|\sum a_{i} \hat{F}\left(g_{i}\right)\right| \leqq\|F\|\left\|\sum a_{i} g_{i}\right\|_{\infty}
$$

for any finite set of $g_{i}$ in $D$, and scalars, $a_{i}$. Thus

$$
\left|\sum a_{i} \hat{x}\left(g_{i}\right)\right| \leqq\|x\|\left\|\sum a_{i} g_{i}\right\|_{\infty} .
$$


But $t^{1 \cdot}$ s reans that $\hat{x}$ satisfies Eberlein's criterion [9] for $\hat{x}$ to be the $\because$ altjes transform of some measure, $m(x)$, on $G$. (The crux of the matter is the measurability of $\hat{x}$. The Fourier-Stieltjes transforms of measures on $B$ which do not come from measures on $G$ will not be measurable with respect to the Haar measure on $D$.)

Thus for each $x$ in $A$ there exists a (necessarily unique) measure $m(x)$

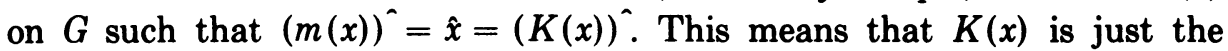
image of $m(x)$ under the natural map of $M(G)$ into $M(B)$ induced by the natural map of $G$ into $B$. Then it will be clear that the map $x \rightarrow m(x)$ is is isometric algebra and order embedding of $A$ into $M(G)$ once we show that the natural map of $M(G)$ into $M(B)$ is an isometric algebra and order embedding. This last fact is undoubtedly well known, but since we have found no proof in the literature we sketch one here which may be new.

4.11.1. Proposition. Let $G$ be a locally compact Abelian group and let $B$ be its Bohr compactification. Then the natural map of $M(G)$ into $M(B)$ is an isometric algebra and order embedding.

Proof. Let $P$ be the $C^{*}$-algebra of almost periodic functions on $G$, so that $B$ is the maximal ideal space of $P[12$, p. 165]. Let $h$ be the Haar measure on $G$, and suppose that $m$ is an element of $M(G)$. Then if we view $P$ as a sub- $C^{*}$-algebra of $L^{\infty}(G,|m|+h)$ (we include the Haar measure so that there is no need to take a quotient algebra of $P$ ), the theorem on the uniqueness of Fourier-Stieltjes transforms tells us that the annihilator in $L(G,|m|+h)$ of $P$ is $\{0\}$. It then follows exactly as in 4.4 that the unit ball of $P$ is dense in the unit ball of $L^{\infty}(G,|m|+h)$ in the $w^{*}$ topology, and so the norm of $m$ as a linear functional on $P$ is the same as the norm of $m$ as an element of $M(G)$. Thus the map of $M(G)$ into $M(B)$ is an isometry.

That the map is an algebra and order isomorphism then follows exactly as in 4.5 and 4.10 .

Alternatively, a proof can be given using Eberlein's characterization of Fourier-Stieltjes transforms [9].

This concludes the proof of Theorem B.

4.13. Observations. There are two facts which we need to note about the above construction. The first is that the smallest closed subgroup, $H$, of $G$ which supports $A$ is $G$ itself. If this were not so, then certain elements of $D$, viewed as characters of $G$, would coincide on $H$, and so would represent the same m.l.f. on $A$, which would contradict the fact that $D$ is a subset of the maximal ideal space of $A$.

Secondly, it will be quite important in $\S 7$ to know that if $h$ is an almost periodic function on $G$, then the usual supremum norm of $h$ is the same as its norm as a linear functional on $A$. This follows from the fact that 
every almost periodic function on $G$ comes from an element of $C(B)$ of the same norm, and the elements of $C(B)$ come from elements of $L^{\infty}(X, m)$ of the same norm. But the supremum norm of elements of $L^{\infty}(X, m)$ is the same as the norm of these elements when they are viewed as linear functionals on $A$.

A question which we have not been able to resolve is whether there are situations in which hypothesis (2) is not true. We note that hypothesis (2) is true in particular if $D$ is either an open subset or a closed subset of the maximal ideal space of $A$. Both cases occur. If $G$ is not discrete, and $A$ is $M(G)$, then $D$ is open in the maximal ideal space of $A$, but is not closed (or dense) $[15$, p. 235]. If $G$ is the group of integers, $Z$, and $A$ is $L\left(Z^{+}\right)$, the elements of $L(Z)$ which are supported on the non-negative integers, then $A$ satisfies hypotheses (1) and (2), and the maximal ideal space of $A$ is the unit disk, whereas $D$ is the unit circle. Thus $D$ is closed but not open in the maximal ideal space of $A$.

5. Necessity of the Tauberian condition. We now begin the discussion of our characterization of group algebras. At first glance one would hope that if one imposed the condition that every m.l.f. of $A$ be $L^{\prime}$-inducing, then this would be a sufficient condition for $A$ to be $L(G)$, or at least a subalgebra of $L(G)$. The purpose of this section is to give an example showing that this is not the case. We will thus need to impose an even stronger condition of $A$ if it is to be $L(G)$.

Let $T$ be the ordinary circle group. Its dual is $Z$, the integers. We show that:

5.1. Theorem. There is a closed subalgebra, $A$, of $M(T)$, properly containing $L(T)$, such that every m.l.f. of $A$ is $L^{\prime}$-inducing. Further, the only m.l.f. of $A$ are those induced by the (continuous) characters of $T$.

Proof. Salem [16, Theorem III] has constructed continuous positive measures, $m$, on $T$ which are purely singular with respect to Haar measure, $h$, on $T$, and whose Fourier-Stieltjes transforms, $m(n)$, are $O\left(|n|^{-\alpha}\right)$ for some $\alpha>0$ ( $n$ is in $Z$ ). Then if we fix some such $m$, and let $r$ be an integer greater than $2 / \alpha$, then $\left(m^{r}\right)^{\wedge}(n)$ will be $O\left(|n|^{-2}\right)$, and so will be in $L(Z)$. But it is an immediate corollary of the Fourier inversion theorem and the Pontrjagin duality theorem that if the Fourier-Stieltjes transform of a finite measure on a group, $G$, is in $L(\hat{G})$, then the measure itself is in $L(G)$. Thus $m^{r}$ is in $L(T)$. Since $m$ is not in $L(T)$, there is some smallest integer, $s$, such that $m^{s}$ is not in $L(T)$ but $m^{2 s}$ is in $L(T)$. Then, if we let $p$ be the purely singular part of $m^{s}, p^{2}$ is in $L(T)$, but $p$ is purely singular with respect to $h$.

Let now $A=L(T, h+p)$. We must show that $A$ is an algebra under *. Then we will show that the only m.l.f. of $A$ are those induced by charac- 
ters $\mathrm{c}^{f} T$, and finally, we will show that each such m.l.f. is $L^{\prime}$-inducing.

'T hrous at we will use the fact that since $p$ and $h$ are mutually singular, every element $x$ of $A$ is uniquely of the form $y+z$ where $y \in L(T, p)$, $z \in L(T, h)=L(T)$, and so $y$ and $z$ are mutually singular. We now show that the convolution of any two elements of $A$ is actually in $L(T)$, which certainly implies that $A$ is an algebra. Now it is well known that the product of two elements of $L(T)$ is again in $L(T)$, as is the product of an element of $L(T, p)$ with an element of $L(T)$. It remains to show that the product of two elements, $x, y$, in $L(T, p)$ is in $L(T)$. Considered as measures, each of them is absolutely continuous with respect to $p$, and so the Cartesian product measure $x \times y$ on $T \times T$ is absolutely continuous with respect to $p \times p$. But if $E$ is a Borel subset of $T$, and if we define

$$
E^{\prime}=\{(r, s): r+s \in E\},
$$

then by definition

$$
(x * y)(E)=(x \times y)\left(E^{\prime}\right) \text { and }(p * p)(E)=(p \times p)\left(E^{\prime}\right)
$$

$[15$, p. 229]. Then if $E$ is of $0 h$-measure, since $p * p \in L(T)$,

$$
(p \times p)\left(E^{\prime}\right)=(p * p)(E)=0,
$$

and so, since $x \times y$ is absolutely continuous with respect to $p \times p$,

$$
(x * y)(E)=(x \times y)\left(E^{\prime}\right)=0 .
$$

Thus $x * y$ is absolutely continuous with respect to $h$, and $x * y \in L(T)$.

We now show that the only m.l.f. of $A$ are those induced by characters of $T$. Let $f$ be any m.l.f. of $A$. Now $f$ cannot be identically zero on $L(T)$, for if it were, then for any $x$ in $A$, since $x * x$ is in $L(T)$, it would follow that $(f(x))^{2}=f(x * x)=0$, and so $f$ would be identically zero on $A$. Then $f$ must agree on $L(T)$ with some m.l.f., $g$, induced by some character of $T$. If we choose $y$ in $L(T)$ such that $f(y)=g(y)=1$, then for any $x$ in $A$

$$
f(x)=f(x) f(y)=f(x * y)=g(x * y)=g(x) g(y)=g(x),
$$

and so $f$ agrees with $g$ on all of $A$.

Finally, since each m.l.f. of $A$ is induced by a character, it is clear from 2.5 that each m.l.f. of $A$ is $L$-inducing, and also satisfies axiom (VIII). Since each m.l.f. of $M(T)$ induced by a character of $T$ satisfies axiom (IX) (3.2), it is clear in view of 1.8 that each m.l.f. of $A$ does also. Thus each m.l.f. of $A$ is $L^{\prime}$-inducing, and the theorem is proved.

We now consider the problem of what condition to impose on $A$ to insure that it is at least a subalgebra of $L(G)$. As indicated above, we will be imposing the condition that every m.l.f. of $A$ be $L^{\prime}$-inducing. Thus $D$ will be locally compact in the $w^{*}$ topology, and the second part of the main theorem will apply. 
A natural additional condition to impose on $A$ is that $A$ be Tauberian, that is, that the elements of $A$ whose Gelfand transforms have compact support be dense in $A$. The reason that this works is that, after carrying out the construction of the main theorem, these elements will correspond to measures on $G$ whose Fourier-Stieltjes transforms have compact support, and so are in $L(\hat{G})$. But, as was seen in the above theorem, this implies that the original measures themselves are in $L(G)$. Thus $A$, under the representation of the main theorem, contains a subset of $L(G)$ which is norm dense in $A$. Since $L(G)$ is norm closed, this means that $A$ must be a subalgebra of $L(G)$. We will see that the Tauberian condition is actually strong enough to insure that $A$ is all of $L(G)$. It is well known that every group algebra is Tauberian [13, 37A].

6. The characterization of group algebras. We are now in a position to prove Theorem $\mathrm{A}$, which we restate here in slightly more detail.

6.1. Theorem A. Let $A$ be a commutative semisimple Banach algebra such that

(1) every m.l.f. of $A$ is $L^{\prime}$-inducing,

(2) $A$ is Tauberian.

Then there exists a locally compact Abelian group, $G$, such that $A$ is isometrically algebra isomorphic to $L(G)$. Further, if $f$ is any fixed m.l.f. of $A$, then this isomorphism can be chosen to be also an order isomorphism of $A$, with order induced by $f$, onto $L(G)$ with its natural order.

Proof. The $D$ of the main theorem is now the entire maximal ideal space of $A$, and so is locally compact, so that hypothesis (2) of the main theorem is automatically satisfied. Hypothesis (1) of the main theorem now becomes simply the hypothesis that $A$ is semisimple. Thus we can apply the second part of the main theorem. If $f$ is the m.l.f. of Theorem 6.1, we obtain a locally compact Abelian group, $G$, such that $A$, with the order induced by $f$, is isometrically algebra and order isomorphic to a closed subalgebra of $M(G)$. As seen at the end of $\$ 5$, hypothesis (2) of Theorem 6.1 implies that $A$ is actually isometrically algebra and order isomorphic to a closed subalgebra of $L(G)$. Identifying $A$ with this subalgebra, what remains to be shown is that $A$ is all of $L(G)$.

Now that we have used the Tauberian condition to insure that $A$ is a subalgebra of $L(G)$, one might suppose that we do not need to use it again. This is true in one case, namely when $D$ is discrete in the $w^{*}$ topology, so that $G$ is compact, for the following reason. It is clear that since $D$ was originally defined as the maximal ideal space of $A$, the Gelfand transforms of the elements of $A$ separate the points of $D$. Since by the main theorem, $D$ is identified with the dual of $G$, the Fourier transforms of the elements 
of the subalgebra $A$ of $L(G)$ separate the points of the dual of $G$. But if is omp ? t, this is a sufficient condition for $A$ to be all of $L(G)[14$, p. 232]. However, if $G$ is not compact, the fact that the Fourier transforms of elements of $A$ separate the points of the dual of $G$ is not a sufficient condition for $A$ to be all of $L(G)$ even if it is also known that $A$ is self-adjoint, that is, $L(G)$ is not a Stone-Weierstrass algebra in general $[14$, p. 239].

In the general case one might suppose that our additional assumption that each m.l.f. be $L^{\prime}$-inducing would be sufficient to insure that $A=L(G)$. We do not know whether this is the case. In our proof that $A=L(G)$ we need to use the Tauberian hypothesis again. The proof falls naturally into two parts. In the first part we show that $A=L(S)$ where $S$ is an open semigroup in $G$, and in the second part we show that $S=G$. We give the first part as a theorem and state it in slightly more generality than needed for our immediate purpose.

6.2. Theorem. Let $G$ be a locally compact Abelian group, and let $A$ be a closed subalgebra of $L(G)$ such that

(1) A separates the points of $\hat{G}$.

(2) Each point of $G$ represents an $L^{\prime}$-inducing m.l.f. of $A$ (but we do not require here that every $m$.l.f. of $A$ is so represented).

(3) $A \cap C_{n}(G)$ is norm dense in $A$.

Then there is an open semigroup, $S$, in $G$, which generates $G$, such that $A=L(S)$.

REMARKS. $C_{\infty}(G)$ is the set of continuous functions on $G$ "vanishing at infinity." Certain elements of $C_{\infty}(G)$ represent elements of $L(G)$, and condition (3) is to be interpreted accordingly. Note that condition (3) is a weakened form of the Tauberian hypothesis, for if $x$ is an element of $L(G)$ whose Fourier transform, $\hat{x}$, has compact support, then by the Fourier inversion theorem, $x=(\hat{x})^{-}$(where denotes the inverse Fourier transform) and so $x$ is represented by a continuous function "vanishing at infinity." By a semigroup, $S$, in a group $G$ we mean simply any subset satisfying $S S \subseteq S$. By $L(S)$ we mean the set of elements of $L(G)$ which are concentrated on $S$. It is well known that for any measurable semigroup $S$ of $G, L(S)$ is a closed subalgebra of $L(G)[14$, p. 233].

Proof of Theorem 6.2. Let $A^{\prime}=A \cap C_{\infty}(G)$. Let

$$
S=\left\{s: x \in G \text {, and } x(s) \neq 0 \text { for some } x \in A^{\prime}\right\} .
$$

Since the elements of $A^{\prime}$ are continuous functions, it is clear that $S$ is open. To show that $S$ is a semigroup, suppose that $s, t \in S$. Then there exist $x, y \in A^{\prime}$ such that $x(s) \neq 0$, and $y(t) \neq 0$. But by hypothesis (2) the constant character 1 represents an $L^{\prime}$-inducing m.l.f. of $A$, and clearly the inclusion of $A$, with order induced by 1 , into $L(G)$, with its usual order, 
is order preserving. Thus, by Proposition $1.8,|x|$ and $|y|$ are in $A$, and so clearly in $A^{\prime}$. Then $|x| *|y|$ is in $A^{\prime}$. But $(|x| *|y|)(s t) \neq 0$ and so st is in $S$.

Since $A$ is norm closed, if we wish to show that $A=L(S)$ it is sufficient to show that $A \subseteq L(S)$, and that $C_{c}^{r+}(S) \subseteq A$, where $C_{c}^{r+}(S)$ is the set of positive real-valued continuous functions of compact support whose support is contained in $S$, since $C_{c}^{r+}(S)$ generates a norm-dense subalgebra of $L(S)$.

Suppose that $A \nsubseteq L(S)$. Then there is an $x$ in $A$ and a measurable set $E$ of strictly positive $x$-measure such that $E \cap S=\emptyset$. Then for $y$ in $A^{\prime}$, $y(s)=0$ for all $s$ in $E$, so

$$
\|x-y\|=\int_{G}|x-y| \geqq \int_{E}|x|>0,
$$

contradicting hypothesis (3). Thus $A \subseteq L(S)$.

Now define $A^{\prime \prime}$ by

$$
A^{\prime \prime}=\left\{x: x \in A^{\prime} \text {, and } x \text { is real }\right\} .
$$

As above, hypothesis (2) and Proposition 1.8 imply that $A^{\prime \prime}$ is a vector sublattice of $C_{\infty}^{r}(G)$, and, also by hypothesis (2) and Proposition 1.8, if $s \in S$ then there exists an $x \in A^{\prime \prime}$ such that $x(s) \neq 0$. We wish to show that any real-valued continuous function on $G$ can be approximated at any two points of $S$ by an element of $A^{\prime \prime}$, so that we will be able to apply Stone's Theorem 1 [17, p. 170], (see also $[13,4 \mathrm{C}]$ ). For this it is sufficient to show that, given any ordered pair, $(s, t)$, of points of $S$, there is an $x$ in $A^{\prime \prime}$ which is different from 0 at $s$, but takes values 0 at $t$. But, given $(s, t)$, since the characters of $G$ separate the points of $G$, we can find a character, $f$, such that $f(s) \neq f(t)$. Then by taking a sufficiently high power of $f$, we can find a character, $g$, such that $g(s)$ and $g(t)$ are in different quadrants of the complex plane. Suppose, for instance, that $\left(\operatorname{Re}^{+} g\right)(s) \neq 0$, but $\left(\operatorname{Re}^{+} g\right)(t)=0$ (it will be obvious how to treat the other cases which may occur). Since $s \in S$, we may find $x$ in $A^{\prime \prime}$ such that $x(s) \neq 0$. Then, since by hypothesis (2) $\bar{g}$ represents an $L^{\prime}$-inducing m.l.f., we can apply 1.8 and 2.7 and write $x$ in the form

$$
x=x_{1} g-x_{2} g+i\left(x_{3} g-x_{4} g\right),
$$

where $x_{i} \geqq 0$ and each $x_{i} \in A^{\prime \prime}$. Since $x(s) \neq 0$, we must have, say, $x_{1}(s)$ $\neq 0$. Of course, $x_{1} g$ is in $A^{\prime}$, and so if we apply hypothesis (2) and Proposition 1.8 again, $\operatorname{Re}^{+}\left(x_{1} g\right)$ is in $A^{\prime \prime}$. But

$$
\left(\operatorname{Re}^{+}\left(x_{1} g\right)\right)(s)=x_{1}(s)\left(\operatorname{Re}^{+}(g)\right)(s) \neq 0
$$

and

$$
\left(\operatorname{Re}^{+}\left(x_{1} g\right)\right)(t)=x_{1}(t)\left(\operatorname{Re}^{+}(g)\right)(t)=0,
$$


so $\operatorname{Re}^{+}\left(x_{1} g\right)$ is the desired element of $A^{\prime \prime}$.

It i. then clear that any continuous real-valued function "vanishing at infinity" on $G$, and 0 outside $S$ can be approximated at any two points of $G$ by an element of $A^{\prime \prime}$. Applying Stone's Theorem 1, we can conclude that any continuous real-valued function "vanishing at infinity" and 0 outside $S$ can be uniformly approximated by elements of $A^{\prime \prime}$.

Now let $z$ be any element of $C_{c}^{r+}(S)$. Let $y$ be any element of $C_{c}^{r+}(S)$ such that $y \geqq z+1$ on the support of $z$, and let $x$ be a positive element of $A^{\prime \prime}$ approximating $y$ uniformly within $1 / 2$, so that $x \geqq z$. Let $y_{n}$ be an element of $A^{\prime \prime}$ approximating $z$ uniformly on $G$ within $1 / n$, and let $x_{n}$ $=\left(y_{n}^{+}\right) \wedge x$. Then $x_{n}$ is in $A^{\prime \prime}$, and $x_{n}$ also approximates $z$ within $1 / n$, since $x \geqq z \geqq 0$. The pointwise limit of the $x_{n}$ is $z$, and since $x \geqq x_{n} \geqq 0$, we can apply the Lebesgue dominated convergence theorem to conclude that $z$ is the norm limit of the $x_{n}$. Since $A$ is closed, $z$ is in $A$. Thus $C_{c}^{r+}(S)$ $\subseteq A$, and $A=L(S)$.

Finally, we need to check that $S$ generates $G$. But if $S$ generated some smaller subgroup, $H$, of $G$, there would be distinct characters of $G$ which agreed on all of $H$, and so represented the same m.l.f. of $A$, contradicting hypothesis (1).

We do not know whether, if condition (3) is omitted, one can prove the above theorem but conclude only that $S$ is a measurable semigroup in $G$.

In the above theorem we used the fact that every character of $G$ represents an $L^{\prime}$-inducing m.l.f. of $A$ to show that $A=L(S)$ for some open generating semigroup, $S$, of $G$. We now use the hypothesis that every m.l.f. of $A$ is $L^{\prime}$-inducing to show that $S=G$.

If $S$ is an open semigroup in $G$, then by a semicharacter of $S$ we mean any not identically zero, continuous, complex-valued function, $f$, on $S$ satisfying $f(s+t)=f(s) f(t)$ and $|f(s)| \leqq 1$ for all elements $s, t$ of $S$. In the same way that for a l.c.a. group $G$ there is a natural bijection between the set of characters of $G$ and the set of m.l.f. of $L(G)$, there is a natural bijection between the set of semicharacters of $S$ and the set of m.l.f. of $L(S)$ [1, Theorem 4.1]. (Their proof is ostensibly for the case in which $S$ is closed and is the closure of its interior, but their proof applies without alteration to the case in which $S$ is open). This bijection is implemented in the same way as that for characters, namely, if $f$ is a semicharacter of $S$, and $x$ is in $L(S)$, then the value at $x$ of the m.l.f. corresponding to $f$ is $\int_{\mathcal{S}} x(s) f(s) d s$, where of course $d s$ is Haar measure.

Every semicharacter $f$ of $S$ has a "polar decomposition," that is, $f$ is of the form $f=p g$ where $g$ is the restriction to $S$ of a character of $G$, and $p$ is a non-negative semicharacter of $S[1$, Theorem 3.1]. By a proper semicharacter of $S$ we will mean a semicharacter which is not the restriction to $S$ of a character of $G$. It is clear from the "polar decompo- 
sition" that for any proper semicharacter, $f$, of $S$, the corresponding "radial part," $p$, cannot be identically equal to 1 , and so there must be points $s, t$ of $S$ such that $p(s) \neq p(t)$, and so such that $|f(s)| \neq|f(t)|$. By Proposition 2.5 this means that $f$ cannot be an $L^{\prime}$-inducing m.l.f. of $L(S)$. We have thus shown

6.3. Proposition. If $G$ is a l.c.a. group and $S$ is a semigroup in $G$ which is open in $G$ then the $L^{\prime}$-inducing m.l.f. of $L(S)$ are exactly those induced by semicharacters of $S$ which are the restrictions to $S$ of characters of $G$. No proper semicharacter of $S$ is $L^{\prime}$-inducing.

In view of this result, in order to prove that $S=G$ it is sufficient to prove

6.4. Theorem. Let $G$ be a l.c.a. group, $S$ any semigroup in $G$, and $s$ a point in the interior of $S$ whose inverse is not in $S$. Then given any complex number $c$, with $0<|c|<1$, there exists a semicharacter $f$ of $S$ such that $f(s)=c$.

We were not able to prove this in general, and our original proof that $S=G$ used results of this kind for special cases together with the structure theory of l.c.a. groups. Since then Andrew M. Gleason has shown us how to prove Theorem 6.4, and has kindly given us permission to include his proof.

Before giving the proof, we remark that Theorem 6.4 also applies to the closed semigroups that are the closure of their interior which were considered by Arens and Singer [1]. Theorem 6.4 says in some sense that their theory is never trivial.

Proof of Theorem 6.4. The proof consists of five reductions, which for logical and notational convenience we give in reverse order as lemmas. Lemma 6.8 is the most crucial of these reductions for it results in a totally ordered group where the theorem can be proved with only minor complications.

6.5. Lemma. Let $G$ be an Archimedean ordered l.c.a. group, $S$ a semigroup in $G$ such that $t \leqq 0$ for every $t$ in $S$, and let $s$ be a point in $S$ whose inverse is not in $S$. Then the conclusion of 6.4 holds.

Proof. By Theorem 8.1.6 of [14], if $G$ is an Archimedean order l.c.a. group, then $G$ is order, group, and topologically isomorphic either with the real line, $R$, or with a discrete subgroup of $R$. If we assume that $G$ is identified accordingly with $R$ or a discrete subgroup of $R$ then the desired semicharacter of $S$ will be

$$
f(t)=\exp ((t / s) \log c) .
$$

6.6. Lemma. Let $G$ be a totally ordered l.c.a. group, $S$ a semigroup in $G$ such that $t \leqq 0$ for all $t$ in $S$, and $s$ a point of $S$ whose inverse is not in $S$. 
Suppose further that $s$ is a strong order unit for $G$, that is, for any $x$ in $G$ there exist integers $m, n$ such that

$$
m s<x<n s .
$$

Then the conclusion of Theorem 6.4 holds.

Proof. Let $H=\{x$ : for all integers $n, s \leqq n x \leqq-s\}$. It is easily checked that $H$ is a closed subgroup of $G$. Let $K=G / H$, and let $F$ be the canonical homomorphism of $G$ onto $K$. Let $P$ be the set of positive elements in $G$, and let $Q=F(P)$. Then $Q$ will be the set of positive elements for an order on $K$ under which $K$ is a totally ordered group. To show this it is sufficient to check that $Q$ is a closed semigroup in $K$ satisfying $Q \cap(-Q)=\{0\}$, $Q \cup(-Q)=K[14$, p. 194]. But this is easily verified using the fact that $P$ satisfies these properties in $G$, and by checking that the preimage of $Q$ in $G$ is exactly $P \cup H$. It is then easy to check that this order on $K$ is Archimedean. Then let $T=F(S), t=F(s)$. Now $F$ is order preserving, so $r \leqq 0$ for any $r$ in $T$. Clearly $T$ is a semigroup in $K$, and since $s$ is not in $H$, $t<0$ and so the inverse of $t$ is not in $T$. Thus we can apply Lemma 6.5 to the triple $(K, T, t)$ to obtain a semicharacter, $g$, of $T$ such that $g(t)=c$. It is then clear that $f=g \circ F$ is the desired semicharacter of $S$.

6.7. Lемма. Let $G$ be a totally ordered l.c.a. group, $S$ a semigroup in $G$ such that $t \leqq 0$ for every $t$ in $S$, and $s$ a point of $S$ whose inverse is not in $S$. Then the conclusion of Theorem 6.4 holds.

Proof. Let $H=\{x$ : there exist integers $m, n$ such that $m s<x<n s\}$. It is easily checked that $H$ is an open, hence closed, subgroup of $G$. The order on $G$ induces a total order on $H$. Let $T=S \cap H$. Then $T$ is a semigroup in $H$ such that $t \leqq 0$ for all $t$ in $T$, and $s$ is a point of $T$ whose inverse is not in $T$. By definition $H$ has the property that $s$ is a strong order unit for $H$. We can thus apply Lemma 6.6 to obtain a semicharacter, $g$, of $T$ such that $g(s)=c$. Let us denote by $f$ the function on $S$ which agrees with $g$ on $T$ and which is 0 off $T$. Then $f$ is continuous since $H$ is both open and closed. We show that $f$ is a semicharacter of $S$. Let $r$ be any element of $S$ not in $T$. Then clearly $r<x$ for any $x$ in $H$. Thus given any $t$ in $S$ we have $t+r \leqq r$ so $t+r$ is not in $T$ and thus $f(t+r)=0=f(t) f(r)$.

6.8. Lemma. Let $G$ be a l.c.a. group, $S$ a semigroup in $G$ such that no nonzero element of $S$ has its inverse also in $S$, and let $s$ be a nonzero point in the interior of $S$. Then the conclusion of Theorem 6.4 holds.

Proof. Let $S^{\prime}$ denote the interior of $S$ with 0 removed if necessary. Then $S^{\prime}$ will be an open semigroup containing $s$, for the interior of a semigroup is a semigroup, and since no nonzero element of $S$ has its inverse in $S$, 
the removal of 0 from the interior of $S$ will not destroy the semigroup property.

Consider the collection, $W$, of semigroups in $G$ which do not meet $S^{\prime}$, and order $W$ by inclusion. Then $W$ is inductively ordered, for if $\left\{P_{\alpha}\right\}$ is a chain in $W$ then $U P_{\alpha}$ is easily seen to be an element of $W$. Applying Zorn's Lemma, let $P$ be a maximal element of $W$. Then $P$ must be closed, since the closure of an element of $W$ is easily seen to be again in $W . P$ must also contain 0 for otherwise we could adjoin 0 to $P$ to obtain a larger element of $W$.

Next, we claim that $P \cup(-P)=G$. Suppose on the contrary that there is an element, $r$, of $G$ such that neither $r$ nor $-r$ is in $P$. Then $P \cup\{r\}$ and $P \cup\{-r\}$ generate larger semigroups than $P$, and so by the maximality of $P$ in $W$ these must both meet $S^{\prime}$. Thus there exist $p, q$ in $P$ and strictly positive integers $m, n$ such that $m r+p$ and $n(-r)+q$ are both in $S^{\prime}$. But then $n p+m q$ is in $S^{\prime}$ since $S^{\prime}$ is a semigroup, and this contradicts the disjointness of $P$ and $S^{\prime}$.

Since $S^{\prime}$ is disjoint from $P$, it is clear from the above that $S^{\prime} \subseteq(-P)$. However we can in fact show that $S \subseteq(-P)$. We first notice that the sum of an element of $S$ with an element in $S^{\prime}$ is again in $S^{\prime}$, for if $r$ is in $S$ and $t$ is in $S^{\prime}$, then $r+S^{\prime}$ is a neighborhood of $r+t$, and so $r+t$ is in the interior of $S$. Also, $r+t$ is not 0 since $t$ is not 0 and no nonzero element of $S$ has its inverse in $S$. Thus $r+t$ is in $S^{\prime}$. Suppose now that for some $r$ in $S, r$ is not in $-P$, so $-r$ is not in $P$. Then $P \cup\{-r\}$ generates a semigroup which must meet $S^{\prime}$, that is, there exist $p$ in $P$ and a positive integer $n$ such that $n(-r)+p$ is in $S^{\prime}$. But since $n r$ is in $S$, this means that $p$ is in $S^{\prime}$ which contradicts the disjointness of $S^{\prime}$ and $P$.

Now let $H=P \cap(-P)$, so that $H$ is a closed subgroup of $G$ disjoint from $S^{\prime}$. Let $K=G / H$, and let $F$ be the canonical homomorphism of $G$ onto $K$. Let $T=F(S), t=F(s), Q=F(P)$. Then $Q$ will be the set of positive elements for a total order on $K$. This is checked as in Lemma 6.6, the preimage of $Q$ being just $P$. It is clear that $T$ is a semigroup in $K$. Since $s$ in in $S^{\prime}, s$ is not in $H$ and so $t=F(s)$ is not 0 . Since $S \subseteq-P$, it follows that $T \subseteq-Q$, that is, $t \leqq 0$ for every $t$ in $T$. Clearly the inverse of $t$ is not in $T$. Thus we can apply Lemma 6.7 to $(K, T, t)$ to ubtain a semicharacter $g$ on $T$ such that $g(t)=c$. It is then clear that $f=g \circ F$ is the desired semicharacter of $S$.

Proof of Theorem 6.4. Let $\bar{S}$ be the closure of $S$ in $G$. Thus $\bar{S}$ is a closed semigroup, and $s$ is an interior point of $\bar{S}$. The inverse of $s$ cannot be in $\bar{S}$ for if $s_{\alpha}$ were a net of elements of $S$ converging to $-s$, then $-s_{\alpha}$ would converge to $s$, and hence so would $-\left(s+s_{\alpha}+s_{a}\right)$. Since $S$ is a neighborhood of $s$, there would be a $\beta$ such that $-\left(s+s_{j}+s_{j}\right)$ is in $S$, so that $-s$ is in $s_{3}+s_{3}+S \subseteq S$, contrary to assumption. 
Let $H=\bar{S} \cap(-\bar{S})$. If $H$ is empty let $K=G$. Otherwise $H$ will be a is ed subgroup of $G$, and we let $K=G / H$. Let $F$ be the canonical homomorphism of $G$ onto $K$, and let $T=F(S), t=F(s)$. Then $T$ is a semigroup in $K$, and since $F$ is an open map, $t$ will be interior to $T$. Then it is easily checked that no nonzero element of $T$ has its inverse in $T$. Finally, $t$ is not 0 , since we saw above that the inverse of $s$ is not in $\bar{S}$. Thus the triple $(K, T, t)$ satisfies the conditions of Lemma 6.8 , and so we can obtain a semicharacter, $g$, of $T$ such that $g(t)=c$. It is then clear that $f=g \circ F$ is the desired semicharacter of $S$.

It is easy to find examples which show that the condition that $s$ be in the interior of $S$ is necessary.

7. The characterization of measure algebras. We now turn to the problem of characterizing measure algebras. Given the results of Theorem $B$, the remaining problem is to insure that every element of $M(G)$ comes from some element of $A$. We must find some condition which can be stated for abstract Banach algebras, and which, after carrying out the construction of Theorem B, will say that every element of $M(G)$ comes from some element of $A$. We show that we can put Eberlein's characterization of FourierStieltjes transforms [9] into such an abstract form.

A slightly weakened form of Eberlein's characterization states that if $c$ is a continuous function on $G$ for which there exists some constant, $k$, such that for any choice of points, $p_{1}, \cdots, p_{n}$, of $G$ and scalars, $a_{1}, \cdots, a_{n}$,

$$
\left|\sum a_{i} c\left(p_{i}\right)\right| \leqq k\left\|\sum a_{i} p_{i}\right\|_{\infty},
$$

where $\left\|\sum a_{i} p_{i}\right\|_{\infty}$ is the supremum norm of the trigonometric polynomial $\sum a_{i} p_{i}$ on $G$, then $c$ is equal to the Fourier-Stieltjes transform of some element of $M(G)$. To put this in abstract form we need only note that $\sum a_{i} p_{i}$ can also be regarded as a linear functional on $M(G)$, and that its norm as a linear functional is the same as $\left\|\sum a_{i} p_{i}\right\|_{\infty}$. With this in mind, and recalling that $G$ is usually only a proper subset of the maximal ideal space of $M(G)$, we make the following definition:

7.1. Definition. Let $A$ be a commutative Banach algebra and let $D$ be an arbitrary subset of the maximal ideal space of $A$ topologized with the $w^{*}$ topology. Then a continuous function, $c$, on $D$ will be said to be a $D$ Eberlein function if it satisfies the following condition: There is a constant, $k$, such that, for any choice of points $p_{1}, \cdots, p_{n}$ of $D$, and scalars, $a_{1}, \cdots, a_{n}$

$$
\left|\sum a_{i} c\left(p_{i}\right)\right| \leqq k\left\|\sum a_{i} p_{i}\right\|
$$

where by $\left\|\sum a_{i} p_{i}\right\|$ is meant the norm of $\sum a_{i} p_{i}$ as a linear functional on $A$.

We can now state and prove our characterization of measure algebras.

7.2. Theorem. Let $A$ be a commutative Banach algebra, and let $D$ be the collection of all $L^{\prime}$-inducing m.l.f. of $A$. Suppose that 
(1) D is a separating family of linear functionals of $A$,

(2) $D$ is locally compact in the $w^{*}$ topology,

(3) every D-Eberlein function is the restriction to $D$ of the Gelfand transform of some element of $A$.

Then there exists a locally compact Abelian group, $G$, whose dual is homeomorphic to $D$, such that $A$ is isometrically algebra isomorphic to $M(G)$. If $f$ is any fixed element of $D$, then this isomorphism can be chosen to be also an order isomorphism of $A$, with the order induced by $f$, onto $M(G)$ with its ordinary order.

Proof. By hypotheses (1) and (2) we can carry out the construction of Theorem B to obtain $A$ embedded in $M(G)$ for some group $G$ whose dual is identified with $D$. Suppose that $m$ is some element of $M(G)$. We wish to show that $m$ is in $A$. Let $\check{m}$ be the inverse Fourier-Stieltjes transform of $m$. Then for any finite set $g_{1}, \cdots, g_{n}$ of points of $D$, and scalars $a_{1}, \cdots, a_{n}$ we have

$$
\left|\sum a_{i} \check{m}\left(g_{i}\right)\right| \leqq\|m\|\left\|\sum a_{i} g_{i}\right\|_{\infty} .
$$

It is now that our second comment in 4.13 becomes important. The function $\sum a_{i} g_{i}$ is almost periodic on $G$, and so, by this comment,

$$
\left\|\sum a_{i} g_{i}\right\|_{\infty}=\left\|\sum a_{i} g_{i}\right\|,
$$

where by the right-hand side we mean the norm of $\sum a_{i} g_{i}$ as a linear functional on $A$. Thus for any choice of points $g_{1}, \cdots, g_{n}$ of $D$, and scalars $a_{1}, \cdots, a_{n}$, we have

$$
\left|\sum a_{i} \check{m}\left(g_{i}\right)\right| \leqq\|m\|\left\|\sum a_{i} g_{i}\right\|,
$$

in other words, $\check{m}$ is a $D$-Eberlein function. Then, by hypothesis (3), $\check{m}$ is the restriction to $D$ of the Gelfand transform of some measure, $n$, in $A$, and so $\check{m}$ is the inverse Fourier-Stieltjes transform of $n$. Then by the uniqueness of inverse Fourier-Stieltjes transforms, $m=n$, and so $m$ is in $A$.

\section{BibLIOGRAPHY}

1. R. F. Arens and I. M. Singer, Generalized analytic functions, Trans. Amer. Math. Soc. 81 (1956), 379-393.

2. G. Birkhoff, Lattice theory, rev. ed., Amer. Math. Soc. Colloq. Publ. Vol. 25, Amer. Math. Soc., Providence, R. I., 1948.

3. - Dependent probabilities and the space $(L)$, Proc. Nat. Acad. Sci. U.S.A. 24 (1938), 154-159.

4. F. T. Birtel, On a commutative extension of a Banach algebra, Proc. Amer. Math. Soc. 13 (1962), 815-822.

5. N. Bourbaki, Eléments de mathématiques, Livre II, Algêbre, Chapitre 1, Structures algébriques, Actualités Sci. Indust. No. 1144, $3^{\mathrm{e}}$ ed., Hermann, Paris, 1958; ibid., Chapitre 6, Groupes et corps ordonnés, Actualités Sci. Indust. No. 1179, Hermann, Paris, 1958.

6. - Eléments de mathématiques, Livre VI, Intégration, Chapitre 5, Intégration des mesures, Actualités Sci. Indust. No. 1244, Hermann, Paris, 1956; ibid., Chapitre 6, 
Intégration vectorielle, Actualités Sci. Indust. No. 1281, Hermann, Paris, 1959.

7. J. Dixmier, Les algebras d'operateurs dans l'espace hilbertien, Gauthiers-Villars, Paris, 1957.

8. N. Dunford and J. T. Schwartz, Linear operators, Part I, Interscience, New York, 1958.

9. W. F. Eberlein, Characterizations of Fourier-Stieltjes transforms, Duke Math. J. 22 (1955), 465-468.

10. B. R. Gelbaum, G. K. Kalish and J. M. H. Olmsted, On the embedding of topological semigroups and integral domains, Proc. Amer. Math. Soc. 2 (1951), 807-821.

11. S. Kakutani, Concrete representation of abstract $(L)$-spaces and the mean ergodic theorem, Ann. of Math. (2) 42 (1941), 523-537.

12. L. H. Loomis, An introduction to abstract harmonic analysis, Van Nostrand, New York, 1953.

13. M. A. Rieffel, A characterization of commutative group algebras and measure algebras, Bull. Amer. Math. Soc. 69 (1963), 812-814.

14. W. Rudin, Fourier analysis on groups, Interscience, New York, 1962.

15. Measure algebras on Abelian groups, Bull. Amer. Math. Soc. 65 (1959), 227-247.

16. R. Salem, On sets of multiplicity for trigonometrical series, Amer. J. Math. 64 (1942), 531-538.

17. M. H. Stone, The generalized Weierstrass approximation theorem, Math. Mag. 21 (1947-1948), 167-184.

\section{University OF CALIFornia, Berkeley, California}

